\title{
Wolbachia dominate Spiroplasma in the co-infected spider mite Tetranychus truncatus
}

\author{
Kun Yang ${ }^{1}$, Kang Xie ${ }^{1}$, Yu-Xi Zhu ${ }^{1}$, Shi-Mei Huo ${ }^{1}$, Ary Hoffmann ${ }^{2}$, Xiao-Yue Hong ${ }^{1 *}$ \\ ${ }^{1}$ Department of Entomology, Nanjing Agricultural University, Nanjing, Jiangsu 210095, \\ China \\ ${ }^{2}$ Bio21 Institute, School of BioSciences, The University of Melbourne, Victoria 3010, \\ Australia
}

\section{Running Head: Wolbachia dominate Spiroplasma in spider mite}

*Corresponding author: Xiao-Yue Hong, Tel: +86-25-84395339. E-mail: xyhong@njau.edu.cn

This is the author manuscript accepted for publication and has undergone full peer review but has not been through the copyediting, typesetting, pagination and proofreading process, which may lead to differences between this version and the Version of Record. Please cite this article as doi: 10.1111/imb.12607

This article is protected by copyright. All rights reserved. 


\begin{abstract}
Wolbachia and Spiroplasma are both maternally inherited endosymbionts in arthropods, and they can co-infect the same species. However, how they interact with each other in the same host is not clear. Here we investigate a co-infected Tetranychus truncatus spider mite strain that shares the same genetic background with singly-infected and uninfected strains to detect the impacts of the two symbionts on their host. We found that Wolbachia-infected and Spiroplasma-infected mites can suffer significant fitness costs involving decreased fecundity, although with no effect on lifespan or development. Wolbachia induced incomplete cytoplasmic incompatibility (CI) in T. truncatus both in singly-infected and doubly-infected strains, resulting in female killing. In both females and males of the co-infected spider mite strain, Wolbachia density was higher than Spiroplasma density. Transcriptome analysis of female adults showed that the most differently expressed genes (DEGs) were found between the co-infected strain and both the singly-infected Spiroplasma strain and uninfected strain. The Wolbachia strain had the fewest DEGs compared to the co-infected strain, consistent with the higher density of Wolbachia in the co-infected strain. Wolbachia therefore appears to have a competitive advantage in
\end{abstract}


host mites over Spiroplasma and is likely maintained in populations by cytoplasmic incompatibility despite having deleterious fitness effects.

Key words: Wolbachia, Spiroplasma, spider mite, co-infection, cytoplasmic incompatibility, transcriptome analysis

\section{Introduction}

Maternally inherited endosymbionts are widespread in arthropods, and Wolbachia and Spiroplasma are two well-known endosymbionts with impacts on their hosts. Wolbachia can be responsible for four types of reproductive manipulations on hosts, involving cytoplasmic incompatibility (CI), parthenogenesis, feminization and male-killing (O’Neill et al., 1997). Among them, CI is considered Wolbachia's most common impact on arthropod hosts (Hoffmann and Turelli 1997). These Wolbachia effects assist the spread of the bacterium across hosts, which can be further assisted by benefits provided by Wolbachia on host fitness (Zug and Hammerstein 2015).

Spiroplasma are descendants of Gram-positive bacteria lacking walls that maintain some of the smallest genomes known for self-replicating organisms, and 
they are most often found in association with insects and ticks, including Coleoptera, Diptera, Hemiptera, Homoptera, Hymenoptera, Lepidoptera, Odonata and Acari (Regassa and Gasparich 2006). These bacteria tend to be male-killers in insect hosts (Hurst and Jiggins 2000) although they also have other effects. When Drosophila melanogaster are infected with MSRO Spiroplasma (an isolated strain of Spiroplasma which naturally infects $D$. melanogaster), flies have increased susceptibility to Gram-negative bacterial pathogens (Herren and Lemaitre 2011). However, Spiroplasma also encode a ribosome-inactivating protein (RIP) to protect hosts against parasites and pathogens (Hamilton et al., 2016, Ballinger and Perlman 2017), potentially enhancing host fitness.

In spider mites, Wolbachia are widely distributed, and can be found in many Tetranychidae species such as Tetranychus truncatus, Tetranychus kanzawai, Tetranychus urticae (Zhang et al., 2016). When Wolbachia infects two-spotted mites (Tetranychus urticae), it can induce incomplete CI, with CI strength of the same Wolbachia strain influenced by host genetic backgrounds (Sun et al., 2016, Zhao et al., 2013). In T. urticae, transcriptome analysis shows that Wolbachia affects numerous biological processes, including oxidation-reduction, digestion and detoxification, and processes involving lipocalins (Zhang et al., 2015). However, Spiroplasma infections are not common in spider mites. To date, Spiroplasma has 
only been found in T. truncates and T. urticae in the Tetranychidae (Staudacher et al., 2017, Zhu et al., 2018). No reproductive manipulations induced by Spiroplasma in spider mites have been found.

Symbiont co-infections are common in arthropods such as spider mites, aphids and grasshoppers (Machtelinckx et al., 2012, Lukasik et al., 2013, Wamwiri et al., 2013, Funkhouser-Jones et al., 2015, Moutailler et al., 2016, Zhang et al., 2016, Zytynska et al., 2016, Nguyen et al., 2017). The presence of co-infections in mites and other arthropods raises questions about how such infections are generally maintained. Do multiple infections increase or decrease the extent and type of reproductive effects associated with single infections? Does the presence of multiple infections lead to additional costs on hosts? What is the nature of competitive interactions among symbionts?

Where coinfections have been investigated, they have a range of phenotypic effects; some have no additional impact over single infections, whereas others appear to enhance the effects of single infections or lead to novel phenotypic effects. In two-spotted mites (Tetranychus urticae), Wolbachia induce weak CI, while Cardinium-infected and doubly-infected males cause severe CI (Xie et al., 2016). In pea aphids, protection against natural enemies provided by symbionts is the same in single infections of Rickettsiella and co-infections with Rickettsiella and Regiella 
symbionts (McLean et al., 2018). Also in aphids, the pathogen-protective phenotype conferred by coinfections with the symbionts Rickettsia and Spiroplasma varies among host genotypes, but is not influenced by co-infection with Hamiltonella (Lukasik et al., 2013). Haplo-diploid thrips populations co-infected by Wolbachia and Cardinium induce complete CI. While Wolbachia density in these thrips co-infections is higher than Cardinium density, Wolbachia removal does not impact Cardinium density, suggesting a lack of competition within hosts (Nguyen et al., 2017).

In the spider mite Tetranychus truncates, Spiroplasma and Wolbachia can co-infect the same individual. To investigate phenotypic effects associated with co-infections, we previously established 4 strains in this species, which involved a co-infected strain, two singly-infected strains and one uninfected strain (Zhang et al., 2018). We found mites doubly-infected with Wolbachia and Spiroplasma gained benefits over singly-infected and uninfected T. truncatus. However, spider mites tested in that study were collected from different sites and shared different genetic backgrounds. Drosophila data show that Wolbachia-associated fitness effects can depend on genetic background (Dean 2006; Olsen et al., 2001). To conclusively demonstrate effects of co-infection by Wolbachia and Spiroplasma, a controlled genetic background should therefore be used.

This article is protected by copyright. All rights reserved. 
To control genetic background effects, we here performed backcrossing between the three symbiont-infected strains and one uninfected strain. We obtained four different symbiont-infected strains that shared similar genetic backgrounds and compared symbiont effects on hosts by crossing female adults with 1-day-old and 5-day-old male adults. We considered the following questions: 1) Do Wolbachia induce CI in T. truncatus? 2) Do Spiroplasma influence the CI induced by Wolbachia? 3) Do the two endosymbionts affect the longevity and developmental rate of hosts? 4) Does Wolbachia density or male age influence CI strength? 5) Do Wolbachia and Spiroplasma affect the gene expression of $T$. truncates? 6) Finally, which endosymbiont is in the dominant position, Wolbachia or Spiroplasma?

\section{Results}

\section{Wolbachia and Spiroplasma decrease fecundity of host spider mites}

Using the four new spider mite strains sharing a similar genetic background, we made 16 crosses in all combinations (Fig. 1). In order to clarify effects of endosymbionts on host mite fecundity, we scored egg laying for each mite strain, and found that the uninfected strain had the most eggs, regardless of whether 1-day-old or 5-day-old males were used in crosses (Fig. 1A, 1D). For 1-day-old male crosses, fecundity of the strains differed $\left[\mathrm{F}_{(3,217)}=7.512, P<0.01\right.$, one-way ANOVA] and 
fecundity of the W-S- strain $(33.33 \pm 0.65)$ was significantly higher than that of the other three strains, while egg numbers for W-S+ (Wolbachia negative, Spiroplasma positive) differed significantly from W+S- (Wolbachia positive, Spiroplasma negative) $(P<0.05$, posthoc test); and the coinfected $\mathrm{W}+\mathrm{S}+$ strain had an intermediate egg number that was not significantly different compared with W-S+ and W+S- (Fig. S1B, S1C). For fecundity of crosses with the 5-day-old males (Fig. 1D), we found a similar pattern, with strain differences overall $\left[\mathrm{F}_{(3,226)}=27.857, P<0.001\right.$, one-way ANOVA], and the W-S- strain having the highest fecundity (36.28 \pm 0.92$)$ which was significantly different to that of W-S+ (31.29 \pm 1.05$)$ and $W+S-(26.02 \pm 0.88)(P<0.001$, posthoc test). The $\mathrm{W}+\mathrm{S}+$ strain $(25.64 \pm 0.90)$ and $\mathrm{W}-\mathrm{S}+$ also differed significantly $(P<0.001$, posthoc test), a different pattern compared to that observed in crosses with 1-day-old males. The results indicate that both Wolbachia and Spiroplasma decrease fecundity of host mites, with Wolbachia having a relatively larger fitness cost.

\section{Wolbachia induces strong female-killing CI in T. truncates, while Spiroplasma has little effect on reproduction}

To test whether symbionts had an effect on reproduction, we compared the hatch rate of each crossing combination (Fig. 1B, 1E), and found that when

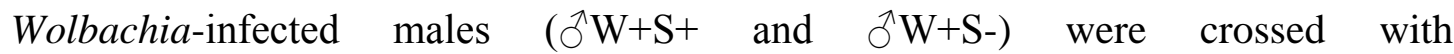
Wolbachia-uninfected females ( $+\mathrm{W}-\mathrm{S}+$ and $\odot \mathrm{W}-\mathrm{S}-)$, there was a significant decline 
in the hatch rate of offspring $\left[\mathrm{F}_{(15,204)}=7.228, P<0.05\right.$, one-way ANOVA $]$. This result showed that Wolbachia induced CI in T. truncates. Furthermore, we found the female offspring number declined in incompatible combinations (Fig. 1C, 1F), pointing to female mortality. There was no impact on reproduction associated with the Spiroplasma infection.

\section{Male age and infected status do not influence Wolbachia-induced CI strength}

To test the impact of male age on inducing CI, we compared hatch rate in crosses involving Wolbachia-infected 1-day-old (Fig. 1B) and 5-day-old (Fig. 1E) males. For 1-day-old male adults, hatchability was similar in the incompatible cross regardless of whether males carried only the Wolbachia infection $(68.73 \pm 4.76 \%)$, or were co-infected $(72.99 \pm 2.99 \%)(P=0.449$, Mann-Whitney U-test). In 5-day-old male incompatible crosses, hatchability was also similar for singly-infected (61.32 $\pm 5.55 \%)$ and co-infected (64.02 $\pm 3.39 \%)$ males ( $P=0.671$, Mann-Whitney U-test). These results also suggest that CI strength induced by Wolbachia is similar regardless of male age (Fig. 1B, 1E), with Mann Whitney $U$ tests indicating no difference in hatch rates for the singly-infected $(P=0.319)$ or coinfected $(P=0.058)$ males.

In females, both endosymbiont densities are higher in the singly-infected strain than in the co-infected strain

This article is protected by copyright. All rights reserved. 
We found that in each developmental stage tested, titer of the symbiont in the singly- infected strain was higher than the same symbiont in the doubly-infected strain $(P<0.001$, Mann-Whitney U-test) (Fig. 2A, B). Both symbionts increased their titer from 1-day-old to 7-day-old adult females, except that Wolbachia in the 3-day-old females from the singly-infected strain had a lower titer than 1-day-old adult females (Fig. 2A). However, in 9-day-old females, the titer in singly-infected strain decreased compared to the titer in 7-day-old adult females, a pattern not seen in the doublyinfected strain.

In the co-infected strain, the titer of Wolbachia was significantly higher than the titer of Spiroplasma in 1-, 3- and 7-day-old females (Figure. 2C); these results indicate that Wolbachia density is higher than Spiroplasma density generally. However, when singly-infected, Wolbachia only had a higher titer than Spiroplasma in 1-day-old and 9-day-old females (Fig. 2D).

\section{In male adults, Wolbachia density and Spiroplasma density are different in singly-infected and co-infected strains}

Spiroplasma in male adults had a significantly higher titer in the singly-infected strain than in the co-infected strain. Except for 1-day-old male adults, the titer of Spiroplasma was significantly higher in the singly-infected strain $(P<0.01$, Mann-Whitney U-tests) (Fig. 2F). In contrast, Wolbachia density in the co-infected 
strain was higher than in the singly-infected strain in four developmental periods ( $P<0.01$, Mann-Whitney $U$-tests). Except in 5-day-old male adults, the singly-infected and co-infected strains had a similar Wolbachia density ( $P=0.49$, Mann-Whitney U-tests) (Fig. 2E). When Wolbachia and Spiroplasma co-infected a male adult, Wolbachia density was significantly higher than Spiroplasma density $(P<0.001$, Mann-Whitney U-test) (Fig. 2G).

\section{Neither Wolbachia nor Spiroplasma affects longevity and developmental rate}

To further clarify the symbionts' effects on host mite fitness, we measured the lifespan (Fig. 3A) and developmental rate of each infected strain (Fig. 3B and C). No significant difference was found in longevity (W+S- vs W-S-, $P=0.113$; W+S- vs $\mathrm{W}+\mathrm{S}+, P=0.984 ; \mathrm{W}+\mathrm{S}-$ vs $\mathrm{W}-\mathrm{S}+, P=0.979 ; \mathrm{W}-\mathrm{S}+$ vs $\mathrm{W}+\mathrm{S}+, P=0.892 ; \mathrm{W}+\mathrm{S}+$ vs W-S-, $P=0.190$; W-S+ vs W-S-, $P=0.154$, Kaplan Meier tests) or developmental rate (male and female) of the strains (see Fig. 3B and 3C).

\section{RNA sequencing and de novo transcriptome assembly}

Eleven T. truncatus transcriptome libraries were sequenced, 3 involving hosts singly-infected by Wolbachia (W+S-), 2 singly-infected with Spiroplasma (W-S+), 3 co-infected with both symbionts $(\mathrm{W}+\mathrm{S}+)$ and 3 without symbiont infections (W-S-). After the trimming of adaptors and filtering for low quality reads, we obtained a total of 568,372,164 clean reads from all 11 libraries (Q30\%>92\%), with an average of 
51,670,197 reads per library. Since there was no published reference genome for $T$. truncatus, we used the complete set of reads to make a de novo transcriptome assembly with Trinity (see methods). After that, Corset (Davidson and Oshlack, 2014) was used to hierarchically cluster the transcripts by reads mapped to the assembled de novo transcriptome and expression pattern (https://code.google.com/p/corset-project/). A total of 57,067 transcripts were assembled, including 47,034 unigenes, encompassing 139 million base pairs (bp). The median length of unigenes was 2276 bp with an N50 of 4681 bp (Table 1). Over 34,231 unigenes were larger than $1 \mathrm{~kb}$ in size. To better evaluate the transcriptional differences between libraries, gene expression levels were divided into six levels according to their fragments per kilobase of exon model per million mapped reads (FPKM) values. Most genes were expressed at low levels in all 11 libraries (Fig. S2).

\section{Gene functional annotation}

In this analysis, 7 databases of gene functional annotation were used. A total of 33,097 genes could be annotated in the NR database (NCBI non-redundant protein sequences) by using Diamond v. 0.8 .22 (with an e-value of 1e-5), covering 70.36\% of the total assembled unigenes (47034). Part of our assembly of genes couldn't be mapped to the NR database, likely linked to misassembly or a lack of representation in the database (Caragata et al., 2017).

This article is protected by copyright. All rights reserved. 
Among the genes mapped to the NR database, $85.5 \%$ of total mapped genes (28215 unigenes) could be matched to the Tetranychus urticae genome (Fig. S3), which showed a high degree of similarity between our assembled T. truncatus transcriptome and the $T$. urticae genome, which is the only genome published for species from the Tetranychidae. T. truncatus and T. urticae are closely related species.

Our assembled unigenes were also annotated to KEGG (Kyoto Encyclopedia of Genes and Genomes) and GO (Gene Ontology) databases (Fig. 4), which were respectively annotated by using KEGG Automatic Annotation Server (with an e-value of 1e-10) and Blast2 GO v2.5. In total, 28498 unigenes could be annotated to the GO database, encompassing $60.59 \%$ of our total assembled unigenes. We identified genes associated with a large range of biological processes, molecular functions and cellular components. From the whole set of contigs, 28,498 were assigned to at least one GO term. The majority of contigs in the biological process category were associated with cellular or metabolic processes. Cell and cellular part were the most frequent classes for genes in cellular component terms, while binding and catalytic activity were the most common terms in the molecular category. The most common KO terms were related to signal transduction.

\section{Transcriptional response to symbionts infection}

This article is protected by copyright. All rights reserved. 
We used a hierarchical clustering heat map to give an overall view of gene expressions of all 4 spider mite lines (Fig. 5). As shown in the map, all 4 groups differed in gene expression patterns, suggesting effects of Wolbachia and/or Spiroplasma infections on expression patterns. Differences were particularly evident when Wolbachia-infected lines were compared to Wolbachia-uninfected lines, suggesting that Wolbachia had larger effects than Spiroplasma on host spider mite expression patterns.

Venn diagrams provide information on differently expressed genes (DEGs) which are particularly evident when $\mathrm{W}+\mathrm{S}+$ is compared to $\mathrm{W}-\mathrm{S}+$ and $\mathrm{W}-\mathrm{S}-$, but less so when $\mathrm{W}+\mathrm{S}+$ is compared to $\mathrm{W}+\mathrm{S}$ - (Fig. 6). In other words, when Wolbachia and Spiroplasma co-infected a single spider mite, Wolbachia effects on gene expression patterns tended to predominate.

DEGs showed differences linked to gene ontology (GO) categories (Fig. 7). When the $\mathrm{W}+\mathrm{S}-$ strain was compared to $\mathrm{W}-\mathrm{S}+$, only genes related to molecular function (MF) categories were upregulated, including genes with scavenger receptor activity and cargo receptor activity (Fig. 7A). These DEGs in the same MF categories were also identified in the comparison of $\mathrm{W}+\mathrm{S}$ - and $\mathrm{W}-\mathrm{S}-$, and in $\mathrm{W}+\mathrm{S}+$ compared to W-S-; DEGs related to scavenger receptor activity and cargo receptor activity were the most abundant in two combinations (Fig. 7C, 7D). However, DEGs in $\mathrm{W}+\mathrm{S}+$ 
compared to $\mathrm{W}-\mathrm{S}+$ showed different ontogenies, involving oxidation-reduction in biological process (BP) (Fig. 7B). Changes in the immunoglobulin complex were uniquely involved in this comparison. Some GO terms were found in both $\mathrm{W}+\mathrm{S}+$ compared to $\mathrm{W}-\mathrm{S}$ - and $\mathrm{W}+\mathrm{S}+$ compared to $\mathrm{W}-\mathrm{S}+$, such as cellulose biosynthetic process and UDP-glucose metabolic process in BP categories, cellular synthase activity and cellulose synthase (UDP-forming) activity in MF categories (Fig. 7B and 7D). Very few genes related to cell component (CC) terms were found in all 4 combinations in GO categories.

\section{Quantitative real-time PCR validation}

With RT-qPCR detection, we confirmed expression differences in 7 random genes, including 10 differently expressed combinations (Fig. 8) and 6 similarly expressed combinations (Fig. 9). For example, two contigs, cluster-2804.19525 and cluster-2804.21232, were predicted to be downregulated by W+S+ compared to W-S-, and further analysis confirmed that expression was significantly lower in $\mathrm{W}+\mathrm{S}+$ (Mann Whitney U tests: $19525-P<0.0001 ; 21232-P<0.001$ ). In total, changes in expression were in good agreement with the RNA-seq results, except for cluster-2804.21764 in W+S- compared to W-S-. Cluster-2804.21764 was predicted to be similarly expressed between $\mathrm{W}+\mathrm{S}$ - and $\mathrm{W}-\mathrm{S}$-; however, our results showed that 21764 was higher in W-S- (Fig. 8K) (Mann Whitney U test: $P<0.0001$ ).

This article is protected by copyright. All rights reserved. 


\section{Interested differently expressed genes between every 4 spider mite strains}

We selected DEGs terms of interest to investigate symbiont-infection interactions in $T$. truncates, including detoxification, immune response, and lipocalins. Most DEGs were detected between the doubly-infected strain and Spiroplasma singly-infected strain or uninfected strain; however, gene expression patterns of the Wolbachia singly-infected strain were similar to the doubly-infected strain (Table 2). Almost all detected genes were homologous to T. urticae genes.

Many spider mites express detoxification genes, such as cytochrome P450 monooxygenases (CYPs), glutathione S-transferases and $\mathrm{ABC}$ transporters. In the present study, detoxification gene expression was largely affected by co-infection of Wolbachia and Spiroplasma, when compared to the uninfected strain or Spiroplasma singly-infected strain. Most CYPs were upregulated in $\mathrm{W}+\mathrm{S}+$; most glutathione S-transferases and ABC transporters were also upregulated in this strain compared to the uninfected strain or the Spiroplasma singly-infected strain.

The lipocalins are a family of proteins which transport small hydrophobic molecules such as steroids, bilins, retinoids, and lipids (Cowan et al., 1990). Contrary to the pattern for detoxification genes, lipocalin genes were downregulated in $\mathrm{W}+\mathrm{S}+$ compared to W-S+. A few differently expressed lipocalin genes were evident in the other comparisons. Two autophagy protein genes were downregulated, and one gene was upregulated in $\mathrm{W}+\mathrm{S}+$ compared to the uninfected strain. Among genes related to 
reproduction, 5 histone-related genes and 3 vitellogenin genes were found differently expressed. We also detected 24 putative immune-related genes affected by symbiont infection, especially in the coinfected strain when compared to the un-infected or singly-infected strain. Lastly there were 33 genes related to lipid metabolic process differently expressed between the strains.

\section{Discussion}

In our experiment, we firstly used a backcrossing experiment to obtain spider mite strains with four similar genetic backgrounds, but differing in symbiont infection status. In comparing the strains, we found different effects of Wolbachia and Spiroplasma on host spider mites. Wolbachia could induce CI in spider mites both in the Wolbachia singly-infected strain and co-infected strains. At the same time, symbiont-infected spider mite strains suffered fitness costs, which lowered fecundity.

In our previous study (Zhang et al., 2018), we found that the spider mites doubly-infected with Wolbachia and Spiroplasma have reproductive and fitness advantages, like higher egg deposition and faster developmental rate, compared to singly-infected and uninfected spider mite strains. Doubly-infected mites could induce incomplete CI, while Wolbachia singly-infected mites could not induce CI. As a result, doubly-infected mites were potentially thought to have a competitive advantage over 
the other mites, helping symbionts spread in the field. However these findings were confounded by the fact that the three symbiont-infected strains were collected from different fields in Shenyang, while uninfected strain was obtained from a tetracycline-treated W-S+ strain. The contrasting findings highlight the importance of controlling genetic background, which has been raised in other systems, such as in the whitefly Bemisia tabaci infected with Rickettsia (Hunter et al., 2016) and in Drosophila melanogaster infected by Wolbachia (Fry et al., 2004; Olsen et al., 2001).

Based on the new results presented here, it is clear that both Wolbachia-infected and Spiroplasma-infected mites have fitness costs, which mainly results in a lower fecundity (Fig. 1A and 1D). These results contrast with the previous study (Zhang et al., 2018), where co-infection was considered to confer host benefits, and the symbiont infections did not have fitness costs. Similar results have been obtained in Nasonia vitripennis, when wasps infected with two Wolbachia strains (wAv and $w \mathrm{Bv}$ ) appeared to produce more offspring when in different genetic backgrounds to the strains used for comparison, whereas no positive fitness effects were found once host genetic background was controlled (Bordenstein and Werren, 2000). Fitness variation, including fecundity and survival, will often largely represent a function of host genetic background (Russell et al., 2018). The costs we have detected for the Wolbachia and Spiroplasma contrast with the effects of a Wolbachia and Spiroplasma 
co-infection in Drosophila melanogaster, where there is no evidence of any benefits or costs when compared to single infections (Montenegro et al., 2006).

Although both endosymbionts are maternally inherited, it is not necessary for them to be beneficial to hosts to spread when they cause cytoplasmic incompatibility (Caspari and Watson, 1959, Hoffmann and Turelli, 1997). Wolbachia induces CI in Tetranychus truncates, although the strength of CI is relatively weak (Fig. 1B and 1E) In a species related to Tetranychus truncates studied here, the two-spotted mite Tetranychus urticae, Wolbachia can also induce incomplete CI (Zhao et al., 2013, Xie et al., 2016). The CI in that species was not affected by age (Sun et al., 2016), which is also the case in the present study, as the hatchability of incompatible crosses with 5-day-old males and 1-day-old males was similar (Fig. 1B and 1E). Spiroplasma had little effect on CI, whereas in Drosophila melanogaster the male-killing ability of Spiroplasma (MSRO strain) was lowered in the presence of Wolbachia (Silva et al., 2012), although different Spiroplasma (NSRO strain) may be unaffected by co-infecting Wolbachia (Goto et al., 2006). To date, cytoplasmic incompatibility is the only Wolbachia-induced reproductive manipulation reported in the Tetranychidae (Breeuwer 1997, Gotoh et al., 2003, Zhu et al., 2012).

Through Q-PCR, we found that Wolbachia and Spiroplasma titers were higher in singly-infected strains, and that Wolbachia titer was significantly higher than 
Spiroplasma in the co-infected strain, except for Wolbachia in male T. truncates (Fig. 3). These results were similar to those found in our former study (Zhang et al., 2018). There was no direct association between CI and Wolbachia density, with Wolbachia density significantly higher in 1-day-old male adults from the co-infected strain (Fig. 2E), but the CI strength being similar between singly and co-infected strains (Fig. 1B). Data from other studies show an inconsistent association between CI strength and Wolbachia density (Duron et al., 2006, Sun et al., 2016) although this relationship may hold in comparisons of the same infection and host species. In mosquitoes, high densities of Wolbachia do not increase the strength of CI or maternal transmission efficiency relative to low Wolbachia densities (Duron et al., 2006). A threshold density of Wolbachia might nevertheless be required to induce CI.

We used transcriptome analysis to compare the DEGs (differently expressed gene) associated with the endosymbionts, which may in turn impact fitness costs and CI for Wolbachia and fitness costs for Spiroplasma (Fig. 1). Previous transcriptome analysis of $T$. urticae indicated that the Wolbachia infection affects numerous biological processes (Zhang et al., 2015) which provides a point of comparison. We matched $85.5 \%$ of expressed genes to mapped $T$. truncates genes. As for T. urticae, $T$. truncates is a polyphagous herbivore feeding on more than 86 plants species (Migeon, 2015), with the expectation that there would be numerous detoxification genes which 
are connected to diet at least in insects (Rane et al., 2019). In T. urticae, genes related to spider mite feeding and detoxification may be influenced by Wolbachia (Zhang et al., 2015), which may indicate a role of the symbiont in host use. In many insects, microbial symbionts can promote the pest status of certain insect species by detoxifying plant allelochemicals (van den Bosch and Welte, 2017). Here we found DEGs involving a set of genes related to detoxification, particularly in $\mathrm{W}+\mathrm{S}+$ (Table 2). This raises the issue of whether Wolbachia might assist in detoxification. Unlike Wolbachia in T. urticae, Wolbachia in T. truncates only influenced two ABC transporter genes (Cluster-2804.16024, Cluster-2804.10578), which suggests a contrasting pattern in these species particularly as $\mathrm{W}+\mathrm{S}-$ of $T$. truncates showed a similar pattern of expression effects to the doubly-infected strain.

Although Wolbachia and Spiroplasma both reduced the fecundity of $T$. truncates, no DEGs related to oogenesis were found between the Wolbachia infected strain and uninfected strain. This was also the case in T. urticae (Zhang et al., 2015). However in the current study some DEGs related to spider mite reproduction, including histones, highly alkaline proteins found in eukaryotic cell nuclei that package and order the DNA into structural units called nucleosomes (Cox et al., 2005). Many histone variants are related to reproduction, and H3.3 is a conserved histone variant that is structurally very close to its canonical counterpart also related 
to reproduction (Orsi et al., 2009). We observed five histone genes related to reproduction with expressions affected by symbiont infection, most of them were found differentially expressed between doubly-infected strain compared to uninfected strain or Spiroplasma singly-infected strain (Table 2). Reproductive effects may also stem from vitellogenins, proteins that are synthesized extra-ovarially and become the major egg yolk protein, vitellin (Hagedorn and Kunkel, 1979). In T. truncates, three vitellogenin genes were differentially expressed between symbiont-infected strains, although these involved both upregulation and downregulation.

In T. urticae, very few genes related to humoral immune and autophagy pathway were differentially expressed by the Wolbachia infection (Zhang et al., 2015), while in present study, only one gene (cluter-2804.3054) related to immune response was induced by Wolbachia. However, co-infection of both Wolbachia and Spiroplasma influenced more putative immune related genes, and most of genes were upregulated, suggesting that it may be worth exploring the role of co-infection of Wolbachia and Spiroplasma on protection from pathogenic bacterial infection which may help explain why co-infection of Wolbachia and Spiroplasma are widespread in nature (Zhang et al., 2018).

Wolbachia dominate Spiroplasma in Tetranychus truncates, as Wolbachia induced reproductive manipulation (CI) and Wolbachia density was significantly 
higher than Spiroplasma density in female and male co-infected adults. Moreover, gene expression patterns of the Wolbachia singly-infected strain were similar to the doubly-infected strain, which showed a stronger effect of Wolbachia in the co-infected strain (Table 2). While co-infection of Wolbachia and Spiroplasma could protect host mites from pathogenic bacteria, it is still unclear why Spiroplasma persist in natural populations when their effects are dominated by Wolbachia.

\section{Experimental Procedures}

\section{Collection and cultivation of Tetranychus truncatus}

All four tested spider mite lines used in this experiment were derived from different sites of Shenyang, Liaoning Province, China. When spider mites transited into the laboratory, isofemale lines of each single strain were established. As spider mites are haplo-diploid, all individuals of one strain are offspring of a single mother. All spider mites in the laboratory were reared on leaves of kidney bean (Phaseolus vulgaris L.). Bean leaves were placed on a water-saturated sponge mat in Petri dishes (dia. 9) at $25 \pm 1^{\circ} \mathrm{C}, 60 \%$ humidity and under L16-D8 conditions.

\section{Detection of Wolbachia and Spiroplasma in T. truncatus}

To detect infection of Wolbachia and Spiroplasma in laboratorial mites, we amplified the Wolbachia surface protein (wsp) gene of Wolbachia with primers 
wsp-F1 and wsp-R1 (Baldo et al., 2006) and the 16s rRNA gene of Spiroplasma with primers of Spits-J04 and Spits-N55 (Jaenike et al., 2007), respectively. Genomic DNA was extracted using TaKaRa MiniBEST Universal Genomic DNA Extraction Kit Ver. 5.0 (TaKaRa BIO INC, Japan). Each reaction was carried out on a Veriti Thermal Cycler (ABI Biosystems, CA, USA) in a $25 \mu \mathrm{l}$ volume containing $12.5 \mu \mathrm{l} 2 \times$ Taq Master Mix (Thermo Scientific, CA, USA), $1 \mu \mathrm{l}$ primer $(20 \mu \mathrm{mol} / \mathrm{L}$ each), and $2 \mu \mathrm{l}$ DNA extract. Positive $(\mathrm{W}+\mathrm{S}+$ female adult DNA template) and negative controls $\left(\mathrm{ddH}_{2} \mathrm{O}\right)$ were included in PCRs.

\section{Establishment of different symbiont-infected T. truncatus strains with similar} genetic backgrounds

We tested all the $T$. truncatus strains in the laboratory and found three different types of symbiont-infected mite strains: 1) doubly-infected strain (mites infected with both Spiroplasma and Wolbachia, named as $\mathrm{W}+\mathrm{S}+$ ), 2) Wolbachia singly-infected strain (named as W+S-), and 3) Spiroplasma singly-infected strain (named as W-S+). Then we generated a mite strain infected with neither Wolbachia nor Spiroplasma (W-S-) by tetracycline treatment of the W-S+ strain for three generations. To eliminate potential side effects of tetracycline, we cultivated the W-S-strain in the lab for seven generations. To reduce genetic background differences among the strains, we then mated three infected female adults $(\mathrm{W}+\mathrm{S}+, \mathrm{W}+\mathrm{S}-$ and $\mathrm{W}-\mathrm{S}+)$ with the male 
uninfected adults (W-S-) and backcrossed female offspring for seven consecutive generations to generate introgressed lines. After that, we cultured the four strains for five generations before experiments. Prior to every experiment, the infection status of symbionts in each strain was checked with PCR.

\section{Crossing experiments between different spider mite strains}

To test how symbionts influence host mite reproduction, such as cytoplasmic incompatibility (CI) or male-killing (MK), we undertook a crossing experiment, which involved mating 1-day-old female virgins of one strain with 1-day-old and 5-day-old unmated male of all other spider mite strains. In total, this experiment includes 16 crossing combinations, and each combination is named as $q$ (infected status) crossed with $\widehat{\jmath}$ (infected status) (Fig. 1). The male adults were allowed to mate with females for 2 days, and the mated females were then allowed to lay eggs for 5 days. Eggs on leaf disks were checked daily to calculate hatchability. After emergence, we assessed the female proportion (male adults divide female adults) of offspring. Fecundity was measured by the number of eggs laid in 5 days. Fecundity and hatchability data were first tested for normality (Kolmogorov-Smirnov test) and homogeneity of group variances (Levene’s test). When the data followed normal distribution, they were analyzed with a one-way ANOVA with post-hoc Tukey HSD analysis. Since female proportion data does not follow a normal distribution, female 
proportion data were analyzed by a Kruskal-Wallis test and Dunn's test with Bonferroni correction for multiple comparisons. SPSS 19 was used to carry out all statistical tests.

\section{Survival assay}

The impacts of symbionts on host mite longevity were tested. Since male spider mites often died accidentally (mite male adults would reach the margin of leaves and drown), we only measured the longevity of female mites. Firstly, 100 third stationary phase (last larval phase before eclosion) female spider mites were selected from the four spider mite strains, and were collected on a single leaf. After 12 hours, all emerged female adults were removed from the leaves. Eight hours later, 24 spider mites of each strain were divided equally across three new leaves, each leaf containing 8 spider mites. The number of dead spider mites was recorded daily. All females were transferred to new leaves every 3 days, to provide fresh food. Survival curves were compared using the Kaplan-Meier method (Bland and Altman 1998).

\section{Developmental rate assay}

The effects of symbionts on host mite development were assessed. Thirty virgins (which produced males) or thirty mated female adults (which produced male and female offspring, respectively) from each strain were placed on a leaf disk and left to 
lay eggs for 3 hours. Eggs were moved to new small leaf disks, with 24 female eggs and 24 male eggs per disk. The disks were monitored every 8 hours, and the stage of mite was recorded until adulthood. The developmental rate of each stage was then calculated. The total development time of spider mite strains were compared with Mann Whitney tests.

\section{Densities of Wolbachia and Spiroplasma}

The Wolbachia density and Spiroplasma density were measured by quantitative PCR (Q-PCR), carried out on the ABI PRISM 7500 Sequence Detection System (Applied Biosystems, CA, USA). The primers used for quantifying Wolbachia target a 141-bp fragment of wsp (wQF1，5'-GAGCAGCGAATGTAAGCAATC-3', and wQR1, 5'-AATAACGAGCACCAGCATAAAG-3'). The primers used for quantifying Spiroplasma target a 141-bp fragment of Spiroplasma16S rRNA gene (sQF1, 5'-TGTAGTTCTCAGGGA TTGTTTTCTC-3', and sQR1, 5'-CGCTTCCACCATCGCTCTT-3') (Zhang et al., 2018). Primers utilized to amplify mite host single copy gene target a 99-bp fragment of rps18 (ribosome protein S18, RPS18-F, 5'-ACGTGCTGGTGAACTTACCGAAGA-3', and rps18-R, 5'-TGCCTATTCAAGAACCAAAGTGGG-3') (Sun et al., 2010). The $20 \mu \mathrm{l}$ Q-PCR reaction mixture consisted of $10 \mu 12 \times$ SYBRP remix Ex Taq (Vazyme, China), $0.4 \mu 1$ $10 \mathrm{mmol} / \mathrm{L}$ of each primer, $0.4 \mu \mathrm{l} 50 \times \mathrm{ROX}$ Reference Dye,2 $\mu \mathrm{l}$ DNA template, and 
$6.8 \mu \mathrm{H}_{2} \mathrm{O}$ in single wells of a 96-well plate (PE Applied Biosystems, CA, USA). The Q-PCR cycling conditions included one cycle $\left(5 \mathrm{~min}\right.$ at $\left.95^{\circ} \mathrm{C}\right)$ followed by 40 cycles (10 s at $95^{\circ} \mathrm{C}$ and $34 \mathrm{~s}$ at $60^{\circ} \mathrm{C}$ ), and finally one cycle for a melt curve $\left(15 \mathrm{~s}\right.$ at $95^{\circ} \mathrm{C}, 1$ $\min$ at $60^{\circ} \mathrm{C}$ and $15 \mathrm{~s}$ at $\left.95^{\circ} \mathrm{C}\right)$. Each DNA sample was run as three replicates. Standard curves were plotted using a 10-fold dilution series of the DNA samples prepared from plasmid DNA. The plasmid DNA was obtained with the pEASY-T3 vector (TransGen Biotech, Beijing, China). The quality and concentration of all purified standard DNA were measured on a Nanodrop 2000 (Thermo Scientific, MA, USA). Densities of straits were compared with Mann-Whitney U-tests.

\section{Sample preparation for Transcriptome analysis}

\section{RNA quantification and qualification}

Twelve groups of samples were prepared for sequencing, with each group going on to form an independent library. Three groups each of 80 7-day old female adults from four strains were collected, except that the W-S+ strain only had two groups. Total RNA was extracted by using Trizol ${ }^{\circledR}$ protocol according to manufacturer's instructions (Invitrogen, CA, USA), for a total of 6 independent samples, with 3 biological replicates per treatment, except for W-S+. RNA purity was checked using the NanoPhotometer ${ }^{\circledR}$ spectrophotometer (IMPLEN, CA, USA). RNA concentration was measured using Qubit ${ }^{\circledR}$ RNA Assay Kit in Qubit ${ }^{\circledR} 2.0$ Flurometer (Life 
Technologies, CA, USA). Sample degradation and contamination were checked by running a portion of the samples on a standard non-denaturing agarose gel containing bleach (Aranda et al., 2012). RNA integrity was assessed using the RNA Nano 6000 Assay Kit of the Agilent Bioanalyzer 2100 system (Agilent Technologies, CA, USA).

\section{Library preparation for Transcriptome sequencing}

A total amount of $1.5 \mu \mathrm{g}$ RNA per sample was used as input material for the RNA sample preparations. Sequencing libraries were generated using NEBNext ${ }^{\circledR}$ Ultra $^{\mathrm{TM}}$ RNA Library Prep Kit for Illumina ${ }^{\circledR}$ (NEB, USA) following manufacturer’s recommendations and index codes were added to attribute sequences to each sample. Briefly, mRNA was purified from total RNA using poly-T oligo-attached magnetic beads. Fragmentation was carried out using divalent cations under elevated temperature in NEBNext First Strand Synthesis Reaction Buffer ( 5X). First strand cDNA was synthesized using random hexamer primer and M-MuLV Reverse Transcriptase (RNase). Second strand cDNA synthesis was subsequently performed with DNA Polymerase I and RNase H. Remaining overhangs were converted into blunt ends via exonuclease/polymerase activities. After adenylation of 3' ends of DNA fragments, NEBNext Adaptor with hairpin loop structure were ligated to prepare for hybridization. In order to select cDNA fragments of preferentially 250 300 bp in length, the library fragments were purified with AMPure XP system 
(Beckman Coulter, Beverly, USA). Then $3 \mu$ l USER Enzyme (NEB, USA) was used with size-selected, adaptor-ligated cDNA at $37^{\circ} \mathrm{C}$ for 15 min followed by 5 min at 95 ${ }^{\circ} \mathrm{C}$ before PCR. Then PCR was performed with Phusion High-Fidelity DNA polymerase, Universal PCR primers and Index (X) Primer. Finally, PCR products were purified (AMPure XP system) and library quality was assessed on the Agilent Bioanalyzer 2100 system.

\section{Clustering and sequencing}

The clustering of the index-coded samples was performed on a cBot Cluster Generation System using TruSeq PE Cluster Kit v3-cBot-HS (Illumia) according to the manufacturer's instructions. After cluster generation, the library preparations were sequenced on an Illumina Hiseq platform and paired-end reads were generated.

\section{Transcriptome analysis}

\section{Quality control}

Raw data (raw reads) of fastq format were firstly processed through in-house perl scripts. In this step, clean data (clean reads) were obtained by removing reads containing adapter, reads containing ploy- $\mathrm{N}$ and low quality reads from raw data. At the same time, Q20, Q30, GC-content and sequence duplication level of the clean data were calculated. All the downstream analyses were based on clean data with high quality. All eleven Illumina RNAseq datasets were combined in order to assemble a 
more reliable transcriptome, with a total of 568,372,164 paired-end, high quality reads, with an average of 51,670,196 clean reads per replicate.

\section{Transcriptome assembly and gene functional annotation}

Since no reference genome was available for $T$. truncates, a de novo transcriptome assembly was built with Trinity using default parameters. The left files (read1 files) from all libraries/samples were pooled into one big left.fq file, and right files (read2 files) into one large right.fq file. Transcriptome assembly was accomplished based on the left.fq and right.fq with min_kmer_cov set to 2 by default and all other parameters set default (Grabherr et al, 2011). The assembled contigs were annotated with Diamond v0.8.22 (http://github.com/bbuchfink/diamond/) to the NCBI non-redundant (NR), Clusters of Orthologous Groups of proteins (COG), euKaryotic Ortholog Groups (KOG) and Swiss-Prot Protein Sequence database. Diamond v0.8.22 parameters were set with an -e value of 1e-5, 1e-3, 1e-3 and 1e-5, respectively. HMMER 3.0 package was used to annotate contigs with Protein family (Pform) database; parameters were set with an -e value of 0.01 . In addition, assembled contigs were annotated to KEGG Ortholog database (KO) with KEGG Automatic Annotation Server, parameters were set with an -e value of 1e-10. Blast2GO v2.5 (Götz et al., 2008) was used to retrieve Gene Ontologies to annotated transcripts; parameters were set with an -e value of 1e-6.

This article is protected by copyright. All rights reserved. 


\section{Quantification of gene expression levels}

Gene expression levels were estimated by RSEM (Li and Dewey, 2011) for each sample, clean data were mapped back onto the assembled transcriptome obtained by Trinity as a reference sequence and read count for each gene was obtained from the mapping results. Parameters of bowtie 2 used in RSEM were mismatch 0.

\section{Differential expression analysis}

Differential expression analysis of two groups was performed using the DESeq $\mathrm{R}$ package 1.10.1 (Kvam et al., 2012). Significantly differently expressed contigs of six combinations (W+S- vs W-S-, W-S+ vs W+S-, W+S+ vs W+S-, W-S+ vs W-S-, $\mathrm{W}+\mathrm{S}+$ vs $\mathrm{W}-\mathrm{S}+$ and $\mathrm{W}+\mathrm{S}+$ vs $\mathrm{W}-\mathrm{S}-$-) were listed, in order to clarify the influence of symbiont infections in T. truncates. DESeq provide statistical routines for determining differential expression in digital gene expression data using a model based on the negative binomial distribution. The resulting $\mathrm{P}$ values were adjusted using the Benjamini and Hochberg approach for controlling the false discovery rate. Genes with an adjusted P-value $<0.05$ found by DESeq were considered differentially expressed.

\section{GO enrichment analysis and KEGG pathway enrichment analysis}

Gene Ontology (GO) enrichment analysis of the differentially expressed genes (DEGs) was implemented by the GOseq R packages based Wallenius non-central hyper-geometric distribution (Young et al., 2010), which can adjust for gene length 
bias in DEGs. GO terms with a corrected $\mathrm{P}$ value less than 0.05 were considered significantly enriched. KEGG (Kanehisa et al., 2008) is a database resource for understanding high-level functions and utilities of the biological system, such as the cell, the organism and the ecosystem, from molecular-level information, especially large-scale molecular datasets generated by genome sequencing and other high-throughput experimental technologies (http://www.genome.jp/kegg/). We used KOBAS (Mao et al., 2005) software to test the statistical enrichment of differential expression genes in KEGG pathways.

\section{Confirmation of differential expression by quantitative real-time PCR}

To confirm the results of RNA-Seq analysis, the expression levels of randomly selected genes were measured by quantitative real time qRT-PCR. The qRT-PCR reactions were performed on the Applied Bio-systems 7500 Real-Time PCR System with the SYBR Premix Ex Taq (Takara Bio, Kyoto, Japan). Primer sequences were designed using Primer Premier 5.0 (Lalitha, 2000).

\section{Conflicts of Interest}

The authors have no conflict among themselves in relation to the article.

\section{Acknowledgments}

We sincerely thank Xue Xia, Ya-Ting Chen and Yue-Ling Song of Nanjing 
Agricultural University, China for their help with the sample collection and species identification. We also thank Dr. Jing-Tao Sun of Nanjing Agricultural University, China for his helpful comments on the manuscript. This study was supported in part by a grant-in-aid for Scientific Research (31672035, 31871976) from the National Natural Science Foundation of China.

\section{References}

Aranda, P. S., LaJoie, D. M. and Jorcyk, C. L. (2012) Bleach gel: a simple agarose gel for analyzing RNA quality. Electrophoresis 33, 366-9.

Baldo, L., J. C., Dunning Hotopp, K. A., Jolley, S. R., Bordenstein, S. A., Biber, R. R., Choudhury, C. et al. (2006) Multilocus sequence typing system for the endosymbiont Wolbachia pipientis. Appl Environ Microbiol 72, 7098-7110.

Ballinger, M. J. and Perlman, S. J. (2017) Generality of toxins in defensive symbiosis: Ribosome-inactivating proteins and defense against parasitic wasps in Drosophila. PLoS Pathog 13, e1006431.

Bland, J. M. and Altman, D. G. (1998) Survival probabilities (the Kaplan-Meier method). Br Med J 317, 1572.

Bordenstein, S. R. and Werren, J. H. (2000) Do Wolbachia influence fecundity in Nasonia vitripennis? Heredity 84, 54-62.

This article is protected by copyright. All rights reserved. 
Breeuwer, J. A. (1997) Wolbachia and cytoplasmic incompatibility in the spider mites Tetranychus urticae and T. turkestani. Heredity 79, 41-47.

Caragata, E. P., Pais, F. S., Baton, L. A., Silva, J. B. L., Sorgine, M. H. F. and Moreira, L. A. (2017) The transcriptome of the mosquito Aedes fluviatilis (Diptera: Culicidae), and transcriptional changes associated with its native Wolbachia infection. BMC Genomics 18, 6.

Caspari, E. and Watson, G. S. (1959) On the evolutionary importance of cytoplasmic sterility in mosquitos. Evolution 13, 568-570

Cowan, S. W., Jones, T. A. and Newcomer, M. E. (1990) Crystallographic refinement of human serum retinol binding protein at 2A resolution. Proteins 8, 44-61.

Cox, M., Nelson, D. R. and Lehninger, A. L. (2005) Lehninger Principles of Biochemistry. San Francisco: W.H. Freeman.

Davidson, N. M. and Oshlack, A. (2014) Corset: enabling differential gene expression analysis for de novo assembled transcriptomes. Genome Biol 15, 410.

Dean, M. D. (2006). A Wolbachia-associated fitness benefit depends on genetic background in Drosophila simulans. Proc Biol Sci 273, 1415-1420.

Duron, O., Labbé, P., Berticat, C., Rousset, F., Guillot, S., Raymond, M. et al. (2006) High Wolbachia density correlates with cost of infection for insecticide resistant Culex pipiens mosquitoes. Evolution 60, 303-314.

This article is protected by copyright. All rights reserved. 
Fry, A. J., Palmer, M. R. and Rand, D. M. (2004) Variable fitness effects of Wolbachia infection in Drosophila melanogaster. Heredity 93, 379-389.

Funkhouser-Jones, L. J., Sehnert, S. R., Martinez-Rodriguez, P., Toribio-Fernandez, R., Pita, M., Bella, J. L. and Bordenstein, S. R. (2015) Wolbachia co-infection in a hybrid zone: discovery of horizontal gene transfers from two Wolbachia supergroups into an animal genome. PeerJ 3, e1479.

Goto, S., Anbutsu, H. and Fukatsu, T. (2006) Asymmetrical interactions between Wolbachia and Spiroplasma endosymbionts coexisting in the same insect host. Appl Environ Microbiol 72, 4805-4810.

Gotoh, T., Noda, H. and Hong, X. Y. (2003) Wolbachia distribution and cytoplasmic incompatibility based on a survey of 42 spider mite species (Acari: Tetranychidae) in Japan. Heredity 91, 208-216.

Götz, S., García-Gómez, J. M., Terol, J., Williams, T. D., Nagaraj, S. H., Nueda, M. J. et al. (2008) High-throughput functional annotation and data mining with the Blast2GO suite. Nucleic Acids Res 36, 3420-3435.

Grabherr, M. G., Haas, B. J., Yassour, M., Levin, J. Z., Thompson, D. A., Amit, I. et al. (2011) Full-length transcriptome assembly from RNA-Seq data without a reference genome. Nat Biotechnol 29, 644-652.

This article is protected by copyright. All rights reserved. 
Hagedorn, H. H. and Kunkel, J. G. (1979) Vitellogenin and Vitellin in insects. Annu Rev Entomol 24, 475-505.

Hamilton, P. T., Peng, F., Boulanger, M. J. and Perlman. S. J. (2016) A ribosome-inactivating protein in a Drosophila defensive symbiont. Proc Natl Acad Sci USA 113, 350-355.

Herren, J. K. and Lemaitre, B. (2011) Spiroplasma and host immunity: activation of humoral immune responses increases endosymbiont load and susceptibility to certain Gram-negative bacterial pathogens in Drosophila melanogaster. Cell Microbiol 13, 1385-1396.

Hoffmann, A. A. and Turelli, M. (1997) Cytoplasmic incompatibility in insects. In O’Neill, S., Hoffmann, A. A. and Werren, J. A. (eds) Influential Passengers: Microorganisms and Invertebrate Reproduction, Oxford University Press: Oxford.

Hunter, M. S., Asiimwe, P., Himler, A. G. and Kelly, S. E. (2016) Host nuclear genotype influences phenotype of a conditional mutualist symbiont. J Evol Biol 30, 141-149.

Hurst, G. D. D. and Jiggins, F. M. (2000) Male-killing bacteria in insects: mechanisms, incidence, and implications. Emerg Infect Dis 6, 329-336. 
Jaenike, J., Polak, M., Fiskin, A., Helou, M. and Minhas, M. (2007) Interspecific transmission of endosymbiotic Spiroplasma by mites. Biol Lett 3, 23-25.

Kanehisa, M., Araki, M., Goto, S., Hattori, M., Hirakawa, M., Itoh, M. et al. (2008). KEGG for linking genomes to life and the environment. Nucleic Acids Res 36, D480-D484.

Kvam, V. M., Liu, P. and Si, Y. (2012) A comparison of statistical methods for detecting differentially expressed genes from RNA-seq data. Am J Bot 99, 248-256.

Lalitha, S. (2000) Primer premier 5. Biotech Software \& Internet Report 1, 270-272.

Li, B. and Dewey, C. (2011) RSEM: accurate transcript quantification from RNA-Seq data with or without a reference genome. BMC Bioinformatics 12, 323-323.

Lukasik, P., Guo, H., Asch, M. V., Ferrari, J. and Godfray, H. C. (2013) Protection against a fungal pathogen conferred by the aphid facultative endosymbionts Rickettsia and Spiroplasma is expressed in multiple host genotypes and species and is not influenced by co-infection with another symbiont. J Evol Biol 26, 2654-2661.

Machtelinckx, T., Leeuwen, T. V., Wiele, T. V. D., Boon, N., Vos, W. H. D., Sanchez, J. A. et al. (2012). Microbial community of predatory bugs of the genus Macrolophus (Hemiptera: Miridae). BMC Microbiol 12, 1-14.

This article is protected by copyright. All rights reserved. 
Mao, X., Cai, T., Olyarchuk, J. G. and Wei, L. (2005) Automated genome annotation and pathway identification using the KEGG Orthology (KO) as a controlled vocabulary. Bioinformatics 21, 3787-3793.

McLean, A. H. C., Parker, B. J., Hrcek, J., Kavanagh, J. C., Wellham, P. A. D., Godfray, H. C. J. (2018) Consequences of symbiont co-infections for insect host phenotypes. J Anim Ecol 87, 478-488.

Migeon, A. (2015) The Jean Gutierrez spider mite collection. ZooKeys 489, 15-24.

Montenegro, H., Petherwick, A. S., Hurst, G. D. D. and Klaczko, L. B. (2006) Fitness effects of Wolbachia and Spiroplasma in Drosophila melanogaster. Genetica 127, 207-215.

Moutailler, S., Moro, C. V., Vaumourin, E., Michelet, L., Tran, F. H., Devillers, E. et al. (2016) Co-infection of Ticks: The Rule Rather Than the Exception. PLoS Neglect Trop D 10, e0004539.

Nguyen, D. T., Morrow, J. L., Spooner-Hart, R. N. and Riegler, M. (2017) Independent cytoplasmic incompatibility induced by Cardinium and Wolbachia maintains endosymbiont coinfections in haplodiploid thrips populations. Evolution 71, 995-1008.

O’Neill, S., Hoffmann, A. A. and Werren, J. A. (eds) (1997) Influential Passengers: Microorganisms and Invertebrate Reproduction. Oxford University Press: Oxford. 
Olsen, K., Reynolds, K. T. and Hoffmann, A. A. (2001) A field cage test of the effects of the endosymbiont Wolbachia on Drosophila melanogaster. Heredity 86, 731-737.

Orsi, G. A., Couble, P. and Loppin, B. (2009) Epigenetic and replacement roles of histone variant H3.3 in reproduction and development. Int J Dev Biol 53, 231-243.

Rane, R. V., Ghodke, A. B., Hoffman, A. A., Edwards, O. R., Walsh, T. K. and Oakeshott, J. G. (2019) Detoxifying enzyme complements and host use phenotypes in 160 insect species. Curr Opin Insect Sci 31, 131-138.

Regassa, L. B. and Gasparich, G. E. (2006) Spiroplasmas: Evolutionary relationships and biodiversity. Front Biosci 11, 2983-3002.

Russell, J. E., Nunney, L., Saum, M. and Stouthamer, R. (2018) Host and symbiont genetic contributions to fitness in a symbiosis. PeerJ 6, e4655.

Silva, N. O., Guenther, L. L., Xie, J. and Mateos, M. (2012) Infection densities of three Spiroplasma, strains in the host Drosophila melanogaster. Symbiosis 57, 83-93.

Sun, J. X., Guo, Y., Zhang, X., Zhu, W. C., Chen, Y. T. and Hong, X. Y. (2016) Effects of host interaction with Wolbachia on cytoplasmic incompatibility in the two-spotted spider mite Tetranychus urticae. Biol J Linn Soc 119, 145-157.

This article is protected by copyright. All rights reserved. 
Sun, W., Jin, Y., He, L., Lu, W. C. and Li, M. (2010) Suitable reference gene selection for different strains and developmental stages of the carmine spider mite, Tetranychus cinnabarinus, using quantitative real-time PCR. J Insect Sci 10, 1-12.

Staudacher, H., Schimmel, B. C. J., Lamers, M. M., Wybouw, N., Groot, A. T. and Kant, M. R. (2017) Independent effects of a herbivore's bacterial symbionts on its performance and induced plant defences. Int. J. Mol. Sci. 18, 182.

van den Bosch, T. J. M. and Welte, C. U. (2017) Detoxifying symbionts in agriculturally important pest insects. Microb Biotechnol 10, 531-540.

Wamwiri, F.N., Alam, U., Thande, P. C., Aksoy, E., Ngure, R. M., Aksoy, S. et al. (2013) Wolbachia, Sodalis and trypanosome co-infections in natural populations of Glossina austeni and Glossina pallidipes. Parasites Vectors 6, 232.

Xie, R. R., Sun, J. T., Xue, X. F. and Hong, X. Y. (2016) Cytoplasmic incompatibility and fitness benefits in the two-spotted spider mite Tetranychus urticae (red form) doubly-infected with Wolbachia and Cardinium. Syst Appl Acarol 21, 1161-1173.

Young, M. D., Wakefield, M. J., Smyth, G. K. and Oshlack, A. (2010) Gene ontology analysis for RNA-seq: accounting for selection bias. Genome Biol 11, R14.

Zhang, Y. K., Chen, Y. T., Yang, K., Qiao, G. X. and Hong, X. Y. (2016) Screening of spider mites (Acari: Tetranychidae) for reproductive endosymbionts reveals links between co-infection and evolutionary history. Sci Rep 6, 27900.

This article is protected by copyright. All rights reserved. 
Zhang, Y. K., Ding X. L., Rong X. and Hong X. Y. (2015) How do hosts react to endosymbionts? A new insight into the molecular mechanisms underlying the Wolbachia-host association. Insect Mol Biol 24, 1-12.

Zhang, Y. K., Yang, K., Zhu, Y. X. and Hong, X. Y. (2018) Symbiont-conferred reproduction and fitness benefits can favour their host occurrence. Ecol Evol 8, 1626-1633.

Zhao, D. X., Zhang, X. F. and Hong, X. Y. (2013) Host-symbiont interactions in spider mite Tetranychus truncates doubly infected with Wolbachia and Cardinium. Environ Entomol 42, 445-452.

Zhu, L. Y., Zhang, K. J., Zhang, Y. K., Ge, C., Gotoh, T. and Hong, X. Y. (2012) Wolbachia strengthens Cardinium-induced cytoplasmic incompatibility in the spider mite Tetranychus piercei Mcgregor. Curr Microbiol 65, 516-523.

Zhu, Y. X., Song, Y. L., Zhang, Y. K., Hoffmann, A. A., Zhou, J. C., Sun, J. T. et al. (2018) Incidence of facultative bacterial endosymbionts in spider mites associated with local environments and host plants. Appl Environ Microbiol 84, e02546-17.

Zug, R. and Hammerstein, P. (2015) Bad guys turned nice? A critical assessment of Wolbachia mutualisms in arthropod hosts. Biol Rev 90, 89-111.

Zytynska, S. E. and Weisser, W. W. (2016) The natural occurrence of secondary bacterial symbionts in aphids. Ecol Entomol 41, 13-26.

This article is protected by copyright. All rights reserved. 
Table 1. Information on de novo assembled genes and genes annotated to different databases for female Tetranychus truncates.

\begin{tabular}{ccc}
\hline Main terms & Gene information and databases & Number \\
\hline De novo assembly & Total Nucleotides & 139357125 \\
genes & N50 & 4681 \\
& N90 & 1646 \\
& Median Length (bp) & 2276 \\
Annotated unigenes & Unigenes larger than 2kb & 25714 \\
& Total number of unigenes & 47034 \\
& NR & 33097 \\
& GO & 28498 \\
& NT & 9078 \\
& KO & 17598 \\
Mapping rate (\%) & SwissProt & 25869 \\
& PFAM & 28323 \\
& KOG & 18301 \\
& Annotated in at least one Database & 10.22 \\
\hline
\end{tabular}

This article is protected by copyright. All rights reserved. 
Table 2. Genes differently expressed by Wolbachia or Spiroplasma infection in

\section{Tetranychus truncates}

\begin{tabular}{|c|c|c|c|c|c|c|c|}
\hline \multirow[b]{2}{*}{ Gene id } & \multirow[b]{2}{*}{ Description } & \multicolumn{6}{|c|}{ Log2 Fold change } \\
\hline & & $\begin{array}{c}\text { W+S+ } \\
\text { vs } \\
\text { W-S+ }\end{array}$ & $\begin{array}{c}\text { W-S+ } \\
\text { vs } \\
\text { W-S- }\end{array}$ & $\begin{array}{c}\text { W-S+ } \\
\text { vs } \\
\text { W+S- }\end{array}$ & $\begin{array}{c}\text { W+S+ } \\
\text { vs } \\
\text { W-S- }\end{array}$ & $\begin{array}{c}\mathrm{W}+\mathrm{S}+ \\
\text { vs } \\
\mathrm{W}+\mathrm{S}-\end{array}$ & $\begin{array}{c}\text { W+S- } \\
\text { vs } \\
\text { W-S- }\end{array}$ \\
\hline \multicolumn{8}{|c|}{ 1. Digestion or Detoxification } \\
\hline Cluster-2804.16063 & Cytochrome P450 2C31-like & 9.1068 & & -20.854 & 9.4098 & & \\
\hline Cluster-2804.15218 & Cytochrome P450 315a1, mitochondrial-like & 8.0879 & & & & & \\
\hline Cluster-2804.9989 & Cytochrome P450 2C31-like & -1.4485 & & & -1.2493 & & \\
\hline Cluster-2804.19836 & Cytochrome P450 302a1, mitochondrial-like & 7.0011 & & -7.7496 & & & \\
\hline Cluster-2804.29491 & Cytochrome P450 3A2-like & -1.8117 & & & & & \\
\hline Cluster-2804.2693 & Cytochrome P450 2J2-like & -2.9173 & & & -1.8888 & & \\
\hline Cluster-2804.14230 & Cytochrome P450 2C31-like & 10.816 & & & 11.119 & & \\
\hline Cluster-2804.1870 & Cytochrome P450 3A16-like & 1.4818 & & & 1.9495 & & \\
\hline Cluster-2804.32745 & Cytochrome P450 2J6-like & & -1.6737 & & -2.2442 & & \\
\hline Cluster-2804.17326 & Cytochrome P450 2C15-like & & & & 1.6068 & & \\
\hline Cluster-2804.24125 & Probable Cytochrome P450 4d14 & & & & -3.5442 & & \\
\hline Cluster-2804.30119 & NADPH--Cytochrome P450 reductase-like & 1.8559 & -2.1815 & -2.3663 & & & \\
\hline Cluster-2804.12797 & NADPH--Cytochrome P450 reductase-like & -8.0893 & 6.5156 & & & & \\
\hline Cluster-2804.23253 & ABC transporter G family member 23-like & 12.606 & & & 12.909 & & \\
\hline Cluster-2804.22450 & ABC transporter $\mathrm{G}$ family member 23-like & 9.8928 & & & 10.196 & & \\
\hline Cluster-2804.33188 & ABC transporter $\mathrm{G}$ family member 23-like & -9.2676 & & & & & \\
\hline Cluster-2804.23533 & ABC transporter G family member 23-like & 8.381 & & & 8.6845 & & \\
\hline Cluster-2804.33190 & ABC transporter $\mathrm{G}$ family member 23-like & 7.0003 & & -7.3219 & & & \\
\hline Cluster-2804.24123 & ABC transporter $\mathrm{G}$ family member 23-like & 6.4392 & & & 6.7432 & & \\
\hline Cluster-2804.25596 & $\mathrm{ABC}$ transporter $\mathrm{G}$ family member 23-like & 7.6067 & & & 7.9086 & & \\
\hline Cluster-2804.18356 & ABC transporter $\mathrm{G}$ family member 23-like & & & & 6.4037 & & \\
\hline
\end{tabular}

This article is protected by copyright. All rights reserved. 


\begin{tabular}{|c|c|c|c|c|c|c|c|}
\hline Cluster-2804.16024 & $\mathrm{ABC}$ transporter $\mathrm{G}$ family member 23-like & & & & 8.424 & & \\
\hline Cluster-2804.10578 & ABC transporter G family member 23-like & & -5.0014 & & -5.8115 & & -6.2048 \\
\hline Cluster-2804.34355 & ABC transporter G family member 22 , partial & & -8.2131 & & & & -6.0555 \\
\hline Cluster-2804.26034 & Glutathione S-transferase Mu 1-like & 8.2286 & & & 8.5327 & & \\
\hline Cluster-2804.10405 & Glutathione S-transferase Mu 1-like & 1.301 & & & & & \\
\hline Cluster-2804.19645 & Glutathione S-transferase Mu 1-like & -1.1569 & & & & & \\
\hline Cluster-2804.25925 & Glutathione S-transferase Mu 1-like & -1.3416 & & & & & \\
\hline Cluster-2804.22715 & Glutathione S-transferase 1, isoform C-like isoform & -1.4679 & & & & & \\
\hline Cluster-2804.11227 & Glutathione S-transferase omega-1-like & & 1.1409 & & 1.7732 & & \\
\hline Cluster-2804.23234 & Glutathione peroxidase 1-like & & & & -7.0922 & & \\
\hline Cluster-2804.36697 & S-formylGlutathione hydrolase-like & 7.3805 & & & 7.683 & & \\
\hline Cluster-2804.13409 & Probable phospholipid hydroperoxide Glutathione & & & & 6.0209 & & \\
\hline & peroxidase isoform $\mathrm{X} 1$ & & & & & & \\
\hline \multicolumn{8}{|l|}{ 2. Lipocalins } \\
\hline Cluster-2804.10646 & Apolipoprotein D-like & -1.5618 & 2.3315 & & & & \\
\hline Cluster-2804.16506 & Apolipoprotein D-like & -2.5738 & 2.6478 & & & & \\
\hline Cluster-2804.23994 & Apolipoprotein D-like & -4.2138 & & & & & \\
\hline Cluster-2804.39826 & Apolipoprotein D-like & 3.7107 & & & 4.4563 & & \\
\hline Cluster-2804.14284 & Apolipoprotein D-like & -2.6151 & & & & & \\
\hline Cluster-2804.4955 & Apolipoprotein D-like & -2.9746 & & & -1.7954 & & \\
\hline Cluster-2804.12557 & Apolipoprotein D-like & -2.4702 & & & & & \\
\hline Cluster-2804.8135 & Apolipoprotein D-like & -1.3026 & & & & & \\
\hline Cluster-2804.12428 & Apolipoprotein D-like & -2.2161 & & & & & \\
\hline Cluster-2804.18399 & Apolipoprotein D-like & -1.8603 & & & & & \\
\hline Cluster-2804.30100 & Apolipoprotein D-like & 1.4812 & & & 1.5823 & & \\
\hline \multicolumn{8}{|c|}{ 3. Autophagy pathway } \\
\hline Cluster-2804.29327 & Autophagy protein 5-like & -10.565 & -1.581 & & -12.134 & & \\
\hline Cluster-2804.28939 & Autophagy protein 5-like & & & & 1.2863 & & \\
\hline Cluster-2804.33629 & Autophagy protein 5-like & & & & -7.2686 & & \\
\hline \multicolumn{8}{|c|}{ 4. Poteintial in reproduction } \\
\hline Cluster-2804.18107 & Histone H3.3 & & -2.2522 & & & & \\
\hline Cluster-2804.22671 & Histone acetyltransferase KAT6B-like & 2.2414 & & -1.7415 & & & \\
\hline Cluster-2804.8385 & Histone-lysine N-methyltransferase & -4.3867 & & & -4.563 & -5.3383 & \\
\hline \multicolumn{8}{|c|}{ SETMAR-like } \\
\hline Cluster-2804.10103 & Sin3 Histone deacetylase corepressor complex & 7.883 & & & 4.7831 & & \\
\hline & component SDS3-like isoform X2 & & & & & & \\
\hline Cluster-2804.24230 & Probable Histone deacetylase 1-B & 1.2223 & & & 1.2738 & & \\
\hline Cluster-2804.19842 & Vitellogenin 4 & & & & 1.8927 & & \\
\hline Cluster-2804.19359 & Vitellogenin-5-like & -1.6396 & 1.8591 & & & & \\
\hline
\end{tabular}


Cluster-2804.19438

Vitellogenin-5-like

5. Immune response

Cluster-2804.32406

Cluster-2804.32405

Cluster-2804.30751

Cluster-2804.26502

Cluster-2804.10580

Cluster-2804.20616

Cluster-2804.36348

Cluster-2804.25100

Cluster-2804.17021

Cluster-2804.18290

Cluster-2804.16594

Cluster-2804.25065

Cluster-2804.10580

\section{Immune response}

Cluster-2804.13527

Cluster-2804.16953

Cluster-2804.27797

Cluster-2804.19655

Cluster-2804.36348

Cluster-2804.9596

Cluster-2804.3054

Cluster-2804.36482

Cluster-2804.2713

Cluster-2804.7370

$$
\text { 2-like }
$$

MHC class I antigen, partial

$$
\begin{gathered}
\text { F-box only protein 47-like } \\
\text { F-box only protein 47-like } \\
\text { F-box only protein 47-like } \\
\text { septin-7-like isoform X2 } \\
\text { palmitoyltransferase ZDHHC17-like } \\
\text { beta-1,3-galactosyltransferase 5-like } \\
\text { filamin-A-like isoform X1 } \\
\text { prostatic acid phosphatase-like } \\
\text { innexin inx2-like } \\
\text { innexin inx2-like } \\
\text { WW domain-containing } \\
\text { oxidoreductase-like } \\
\text { WW domain-containing } \\
\text { oxidoreductase-like }
\end{gathered}
$$$$
\text { Palmitoyltransferase ZDHHC17-like }
$$

$-11.077$

10.568

$-8.6428$

8.8988

$-20.387$

8.5477

$-8.4025$

$-7.1855$

7.6678
$-1.1202$

6.4395

8.7271
11.135

9.2717

$-1.4896$

1.8977

9.5143

1.3481

1.0738

7.8188

$-1.6016$

6.4425

6.7137

1.3626

tyrosine-phosphorylation-regulated kinase

GABA gated chloride channel RDL1 


\section{Figure legends}

Figure 1. Results of crosses between different Tetranychus truncates strains. Egg amount of all combinations involving 1-day-old males (A) and 5-day-old males (D), hatchability of crosses involving 1-day-old males (B) and 5-day-old males (E), female proportion of each combination involving 1-day-old males (C) and 5-day-old males (F). Results are shown as box and whiskers, the same letters above columns represent homogeneous groups in post-hoc tests $(P>0.05)$ following an ANOVA, except for 
female proportion which was analyzed by Kruskal-Wallis tests and Dunn’s tests with

Bonferroni correction for multiple comparisons.

Figure 2. Wolbachia and Spiroplasma density in Tetranychus truncates adults. For female spider mites, symbiont density in singly-infected and co-infected strains is given for Wolbachia (A) and Spiroplasma (B) relative to a host gene. A comparison of Wolbachia and Spiroplasma density is provided for singly-infected (C) and co-infected (D) strains. In male spider mites, symbiont density in singly- and co-infected male adults is presented for Wolbachia (E) and Spiroplasma (F) as well as a comparison of the infections in co-infected male adults (G). Individual data points of the single copy genes $16 S$ rRNA (Spiroplasma), and wsp (Wolbachia) were normalized to the RPS-18 (host) reference gene. Asterisks above points indicate statistically significant differences (Mann-Whitney U test, ${ }^{*} \mathrm{P}<0.05$, ${ }^{* *} P<0.01$, $* * * P<0.001$, NS, not significant).

\author{
Figure 3. Influence of Wolbachia and Spiroplasma on Tetranychus truncates \\ longevity and developmental rate. Survival curves for individual hosts were \\ compared using the Kaplan-Meier method and log-rank test (A). Each strain’s \\ developmental rate is displayed, including for males (B) and females (C). Differences \\ were analyzed using Mann Whitney test (ns, not significant).
}

This article is protected by copyright. All rights reserved. 
Figure 4. GO terms (A) and KEGG terms (B) of female Tetranychus truncates. A.

56 most enriched gene ontology terms, including biological process (red), cellular component (green) and molecular function (blue) catogeries. B. 32 most enriched KEGG terms.

Figure 5. Hierarchical clustering heat map of the gene abundance in the four spider mite strains. Red color means genes highly expressed, blue color means genes weakly expression. Color from red to blue mean Log10 (FPKM+1) from high to low. Figure 6. Venn diagram of DEGs (differently expressed genes) between every comparison combination. A. DEGs of three cytoplasmic incompatibility combinations. B. DEGs of all combinations except for $\mathrm{W}+\mathrm{S}$ - strain compared to W-S+ strain.

Figure 7. Significantly different enriched GO terms. (A) W+S- compared to W-S+; (B) $\mathrm{W}+\mathrm{S}+$ compared to $\mathrm{W}-\mathrm{S}+$; (C) $\mathrm{W}+\mathrm{S}$ - compared to $\mathrm{W}-\mathrm{S}$-; (D) $\mathrm{W}+\mathrm{S}+$ compared to W-S-.

Figure 8. Confirmation of genes differently expression by RT-qPCR. 4 genes differently expressed in 11 different combinations of strains are shown in the graphs. (Mann Whitney U tests, * $\mathrm{P}<0.05$; ** $\mathrm{P}<0.01$; *** $\mathrm{P}<0.001$ ). 
Figure 9. Confirmation of genes not differently expressed by RT-qPCR. 4 genes not differently expressed in 6 combinations of spider strains are shown in the graphs (NS = not significant)

\section{Supplement File}

Figure S1. Design of introgression of spider mites (A), total egg number for each strain in crosses with 1-day-old males (B) and 5-day-old males (C). Columns in different colors stand for different female spider mite strains.

Figure S2. Distribution of FPKM in each Tetranychus truncates sample. The $\mathrm{y}$-axis represents for FPKM number; the $\mathrm{x}$-axis represents different T. truncates samples. FPKM means fragments per kilobase of transcripts per million fragments mapped, different colors represent different FPKM intervals.

Figure S3. Breakdown of gene annotations by organism of origin (comparison by Nr database). 

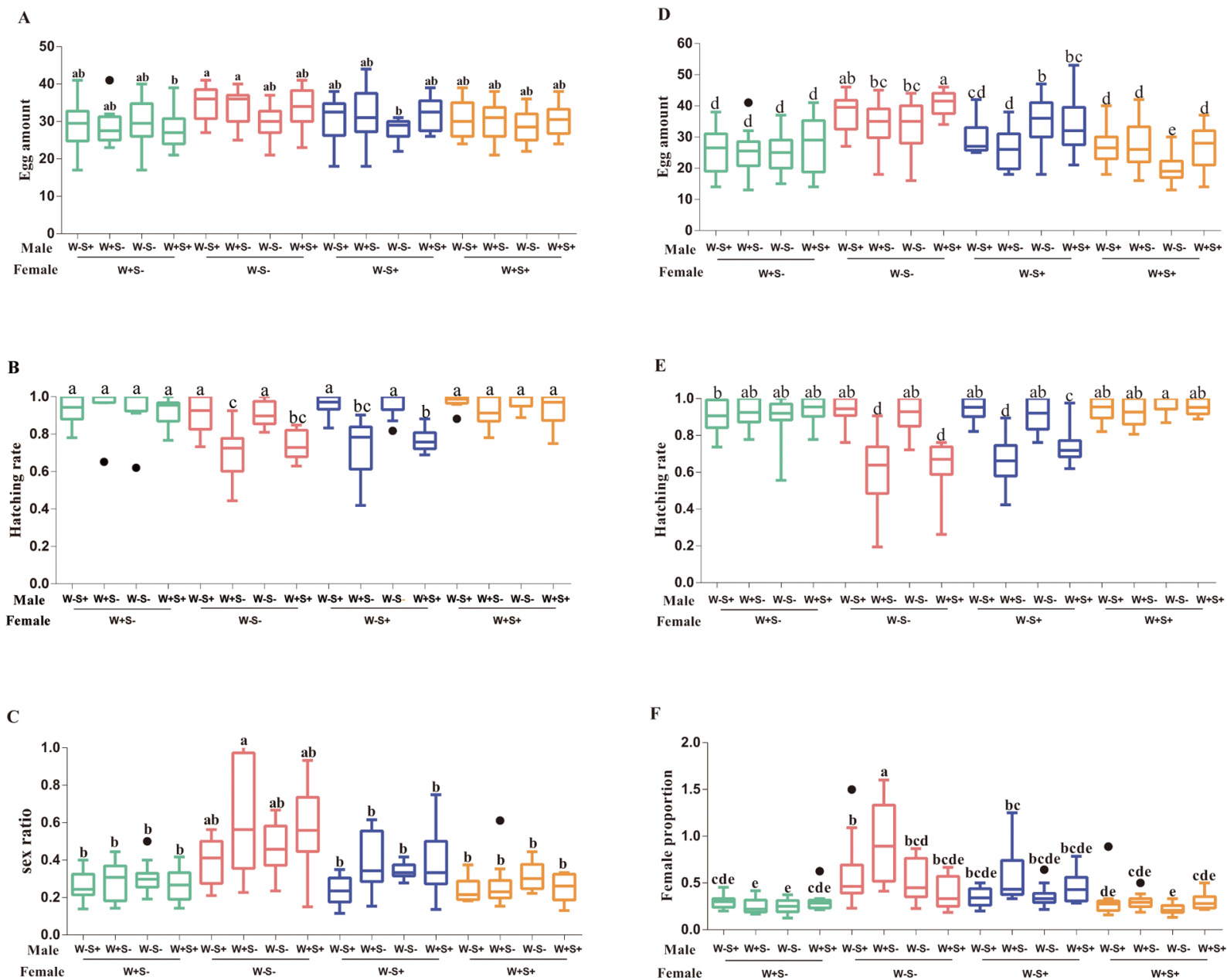

IMB_12607_Figure1.tif 

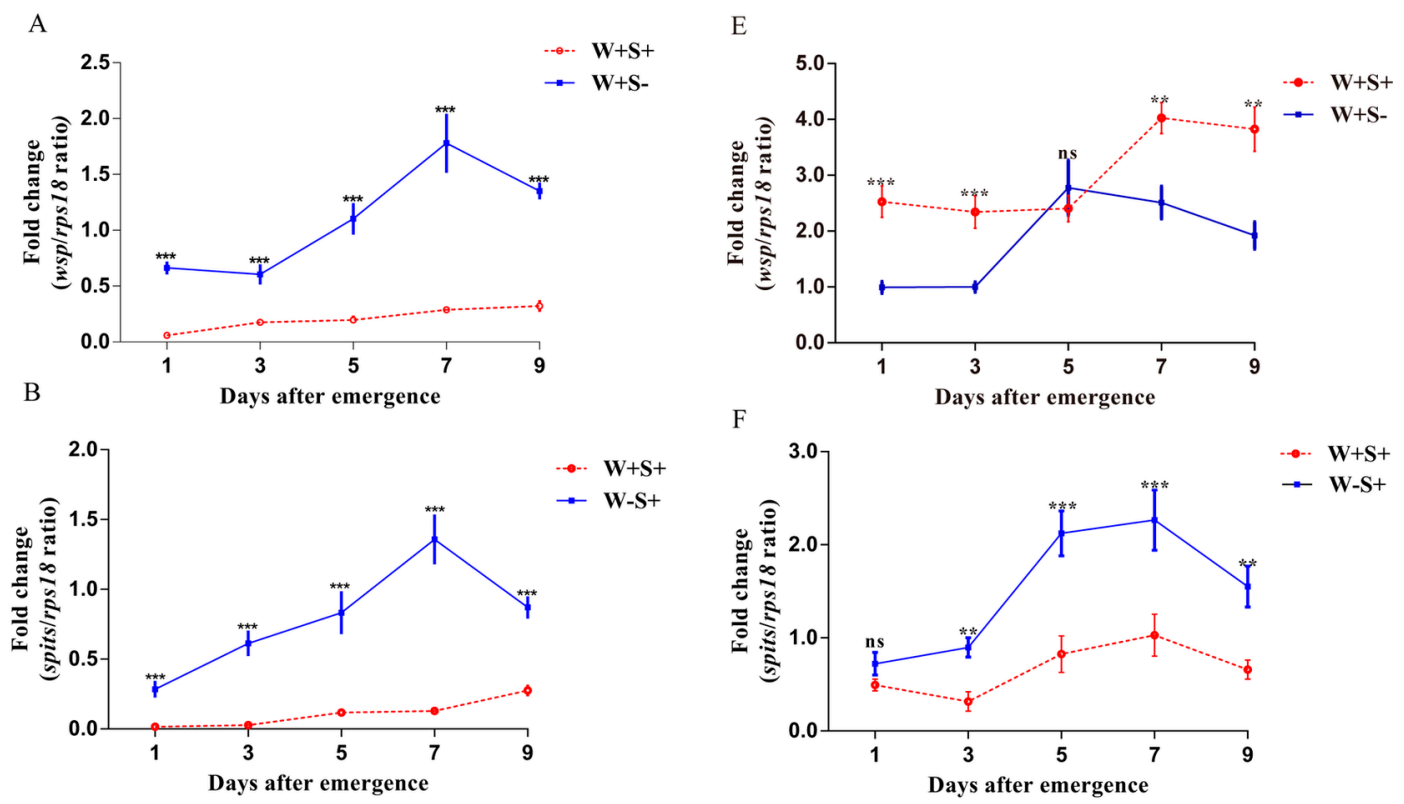

C
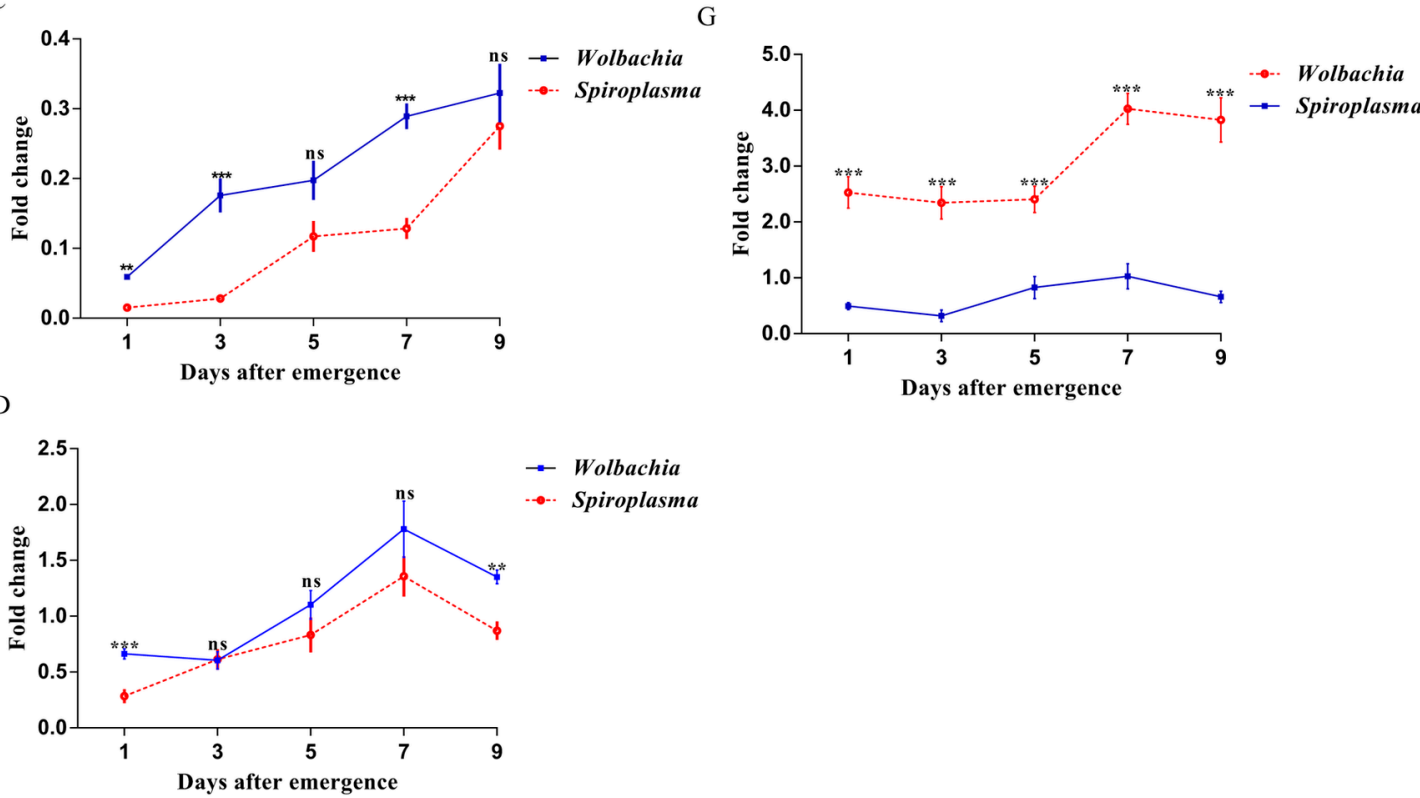

IMB_12607_Figure2.tif

This article is protected by copyright. All rights reserved. 

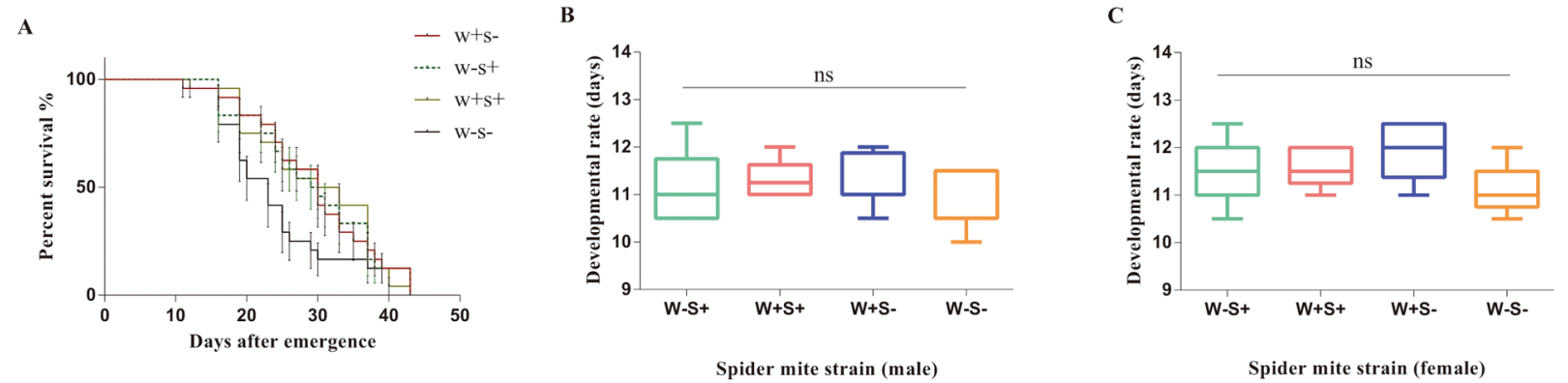

IMB_12607_Figure3.tif

This article is protected by copyright. All rights reserved. 


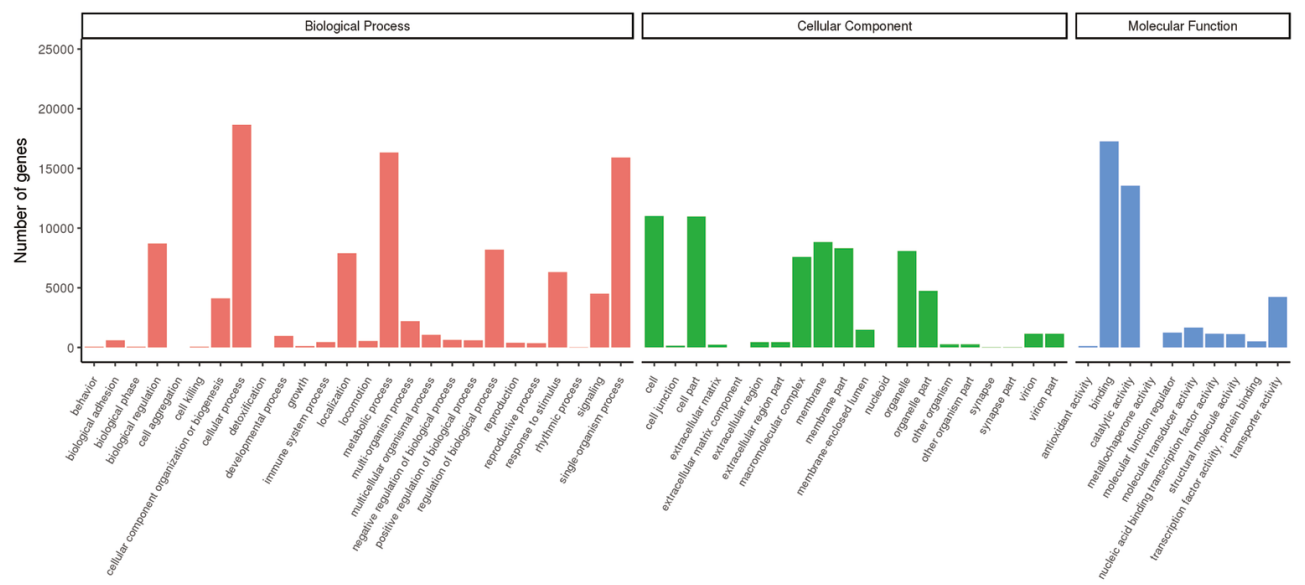

B

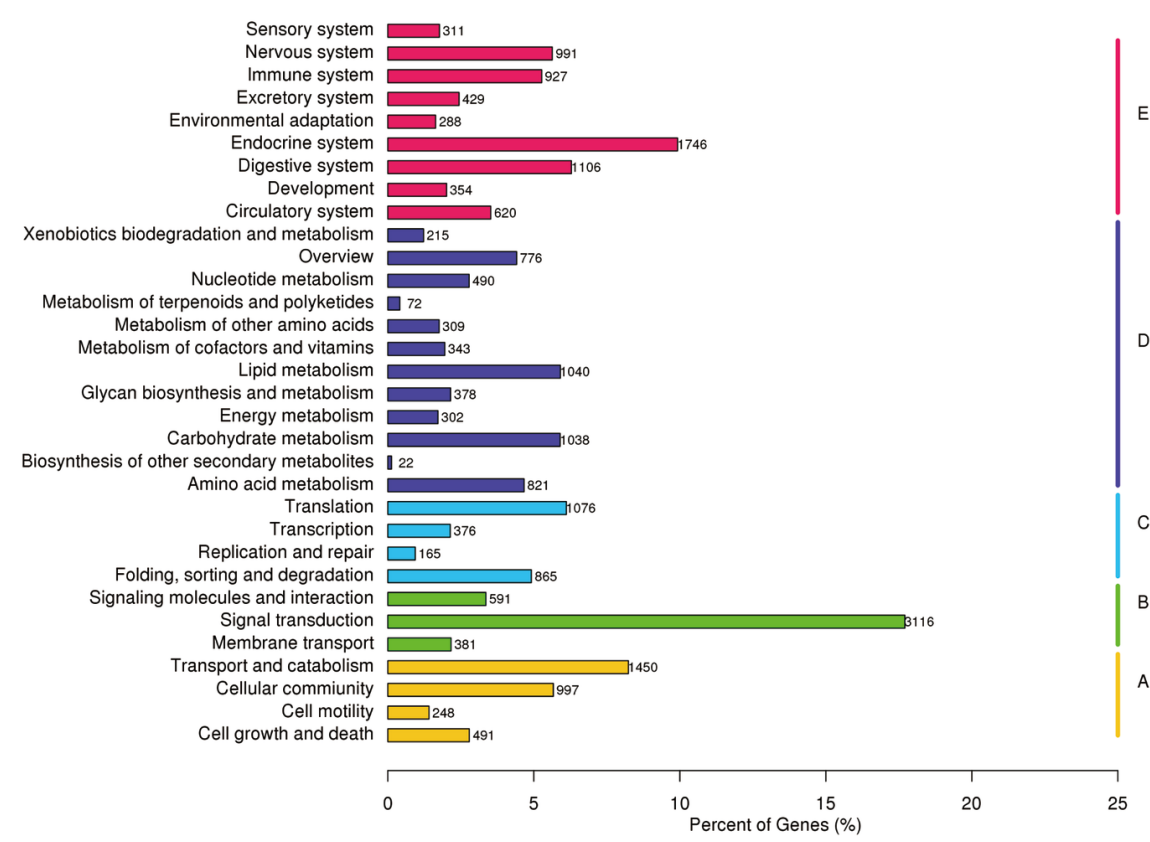

IMB_12607_Figure4.tif

This article is protected by copyright. All rights reserved. 


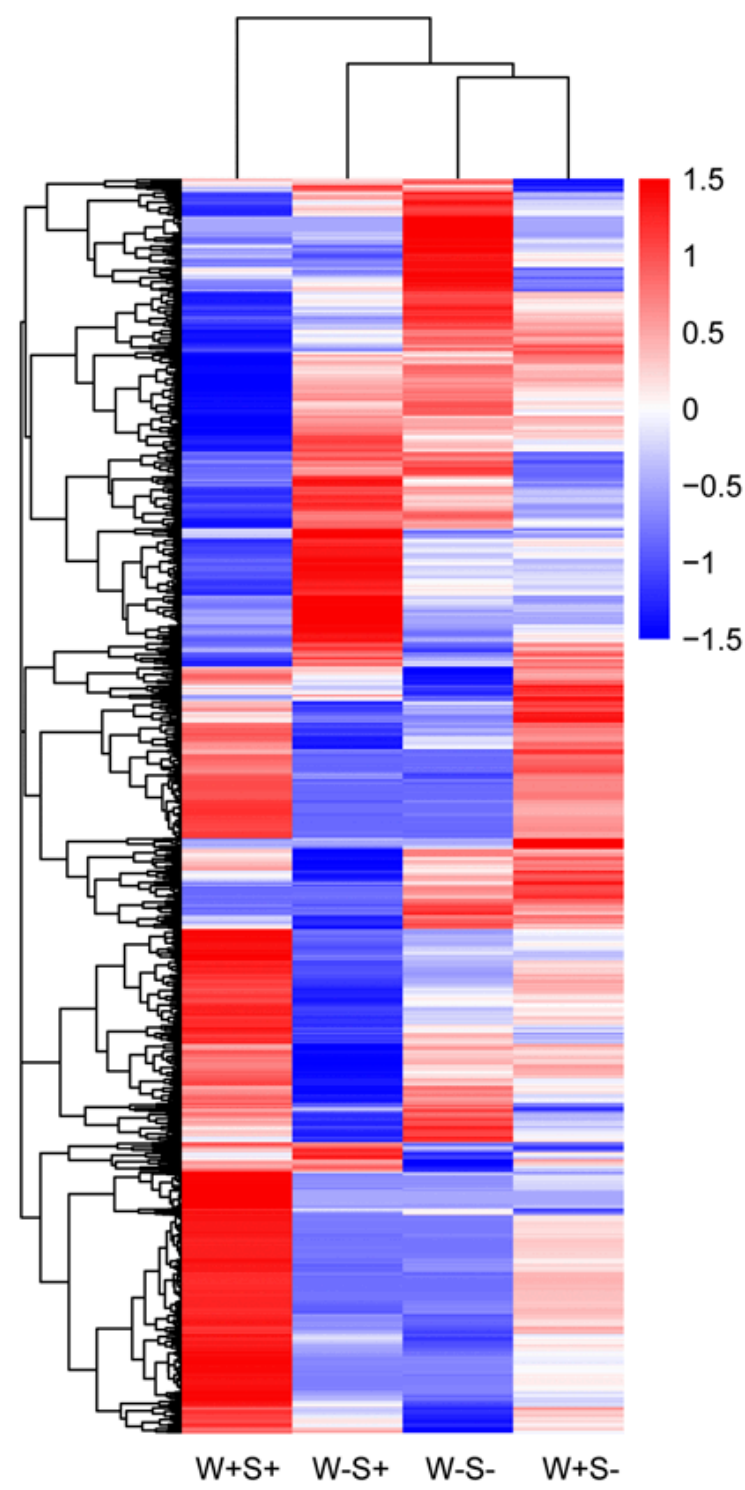

IMB_12607_Figure5.tif

This article is protected by copyright. All rights reserved. 


\section{7}

556

18

15

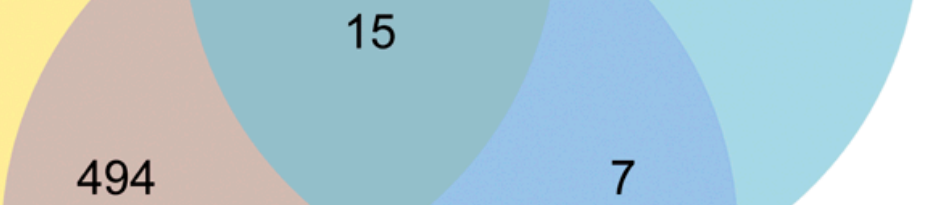

750

$\mathrm{W}+\mathrm{S}+$ vs W-S+

\section{W+S+ vs W-S-}

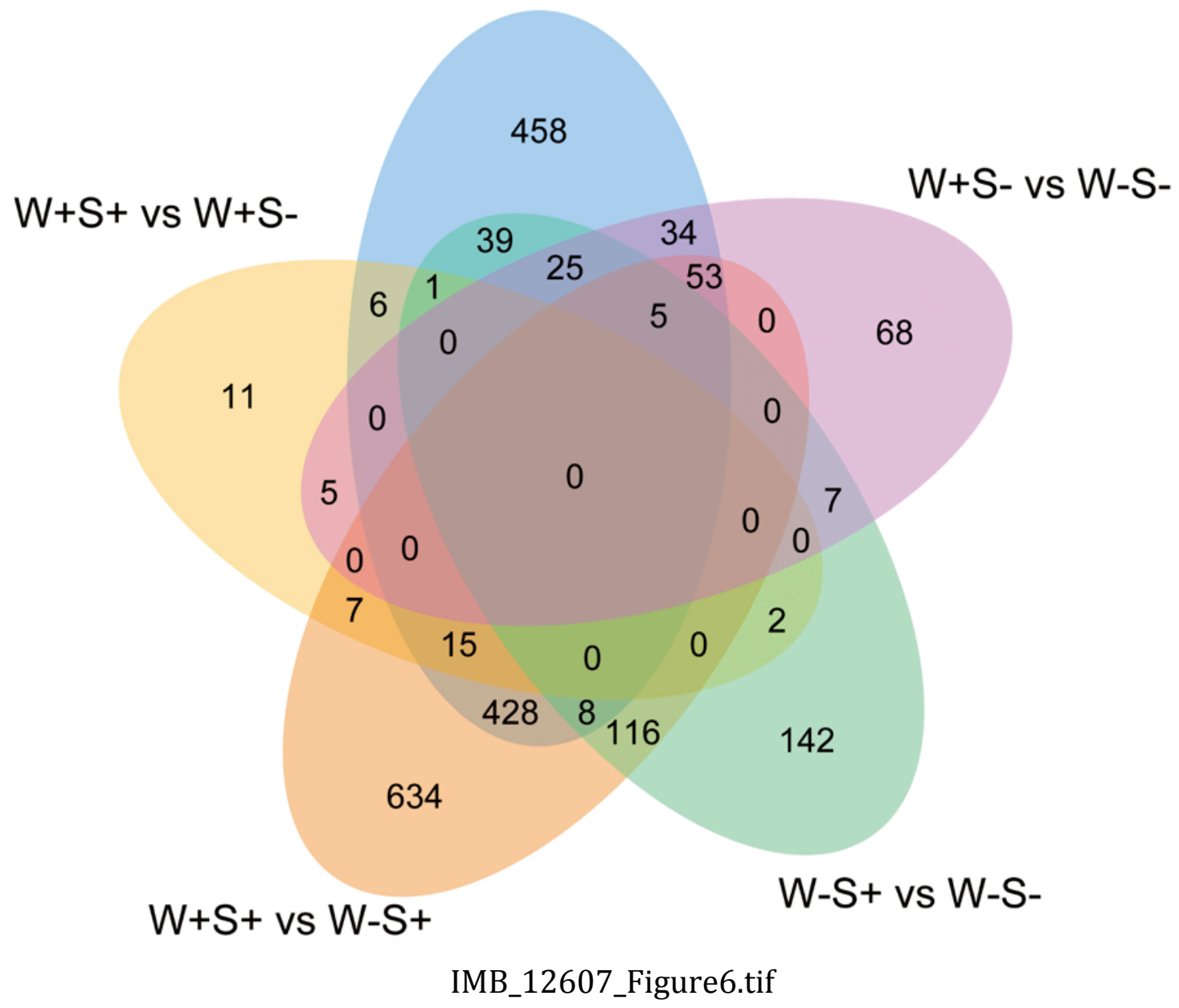

This article is protected by copyright. All rights reserved. 

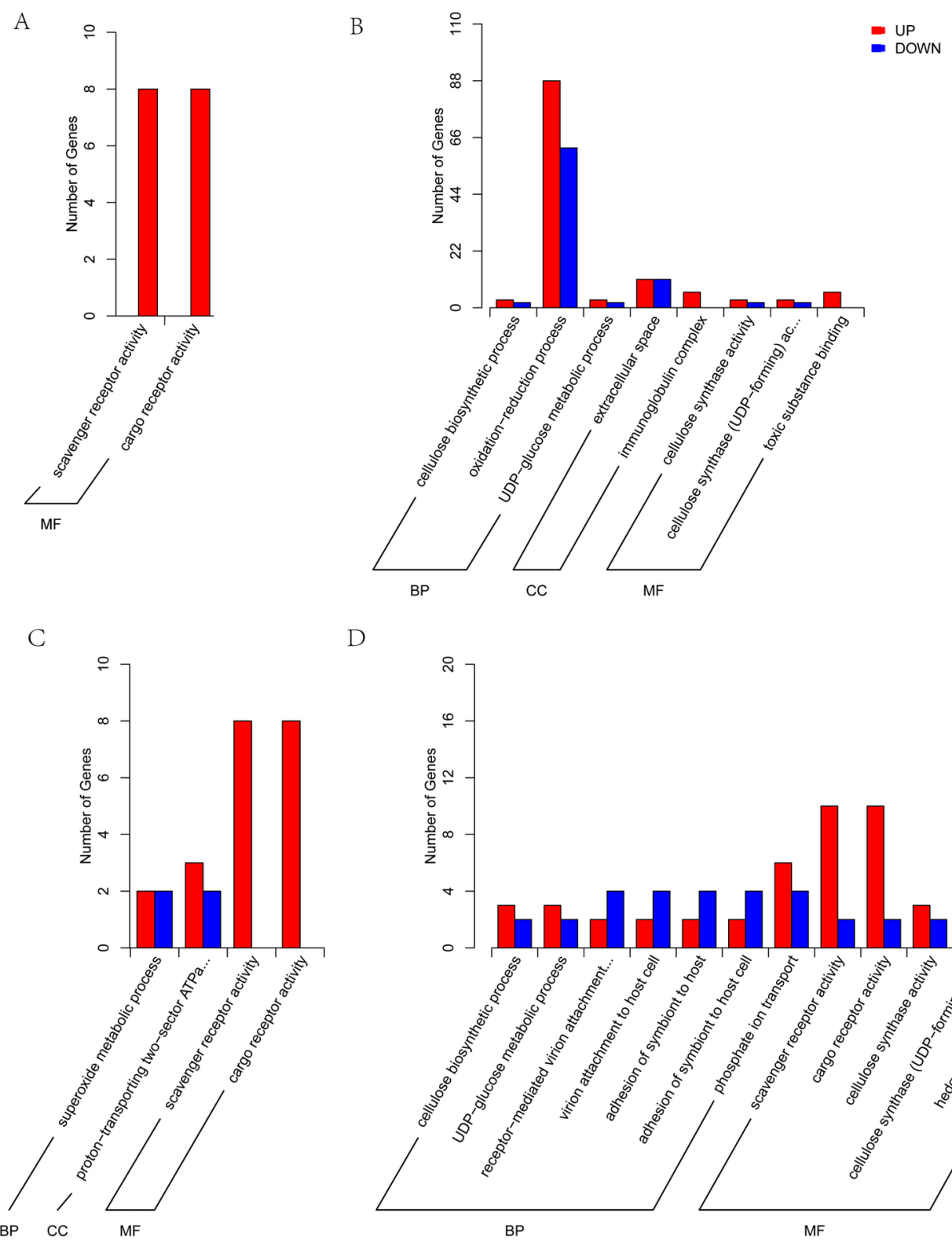

D

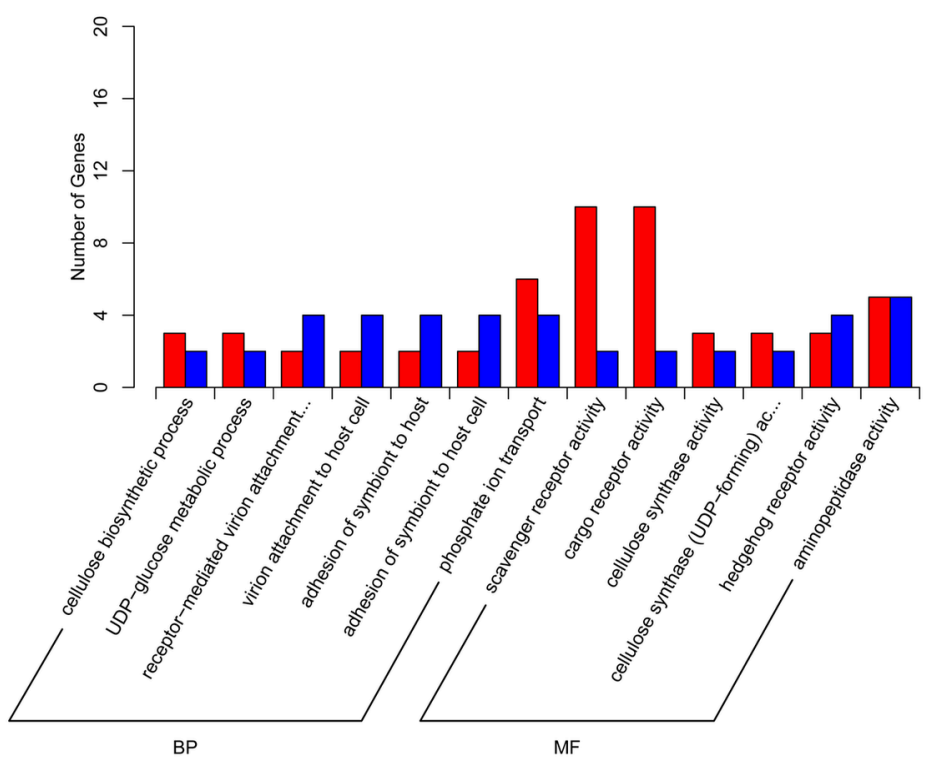

IMB_12607_Figure7.tif 

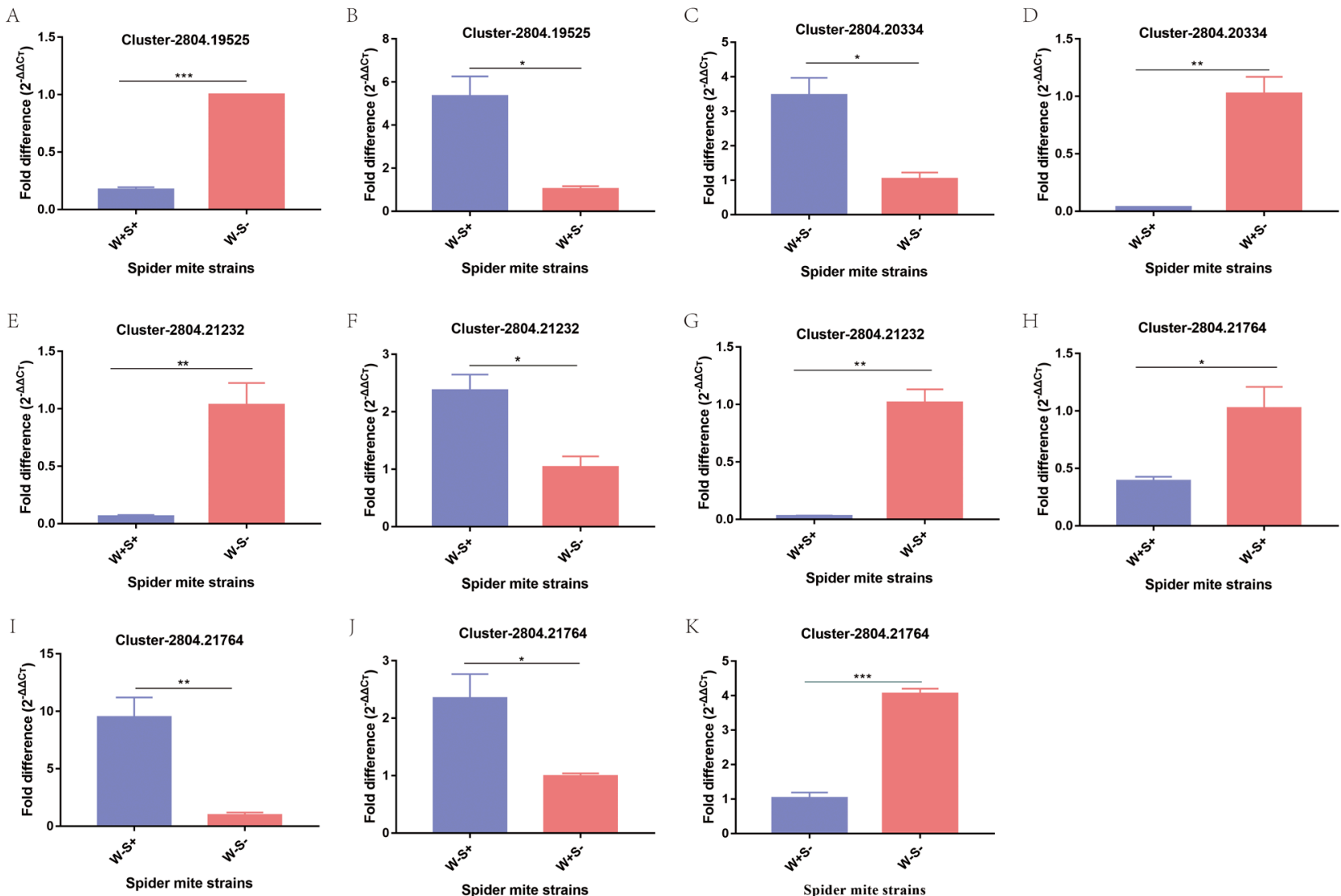

IMB_12607_Figure8.tif 
A

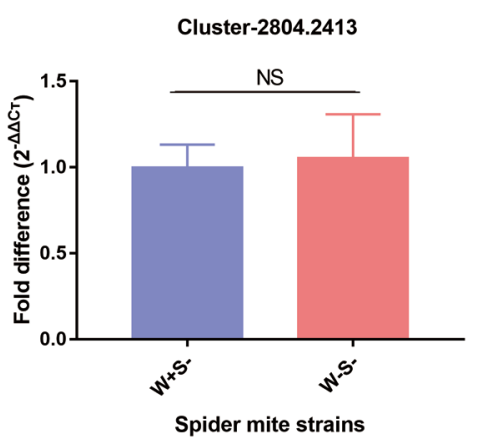

D

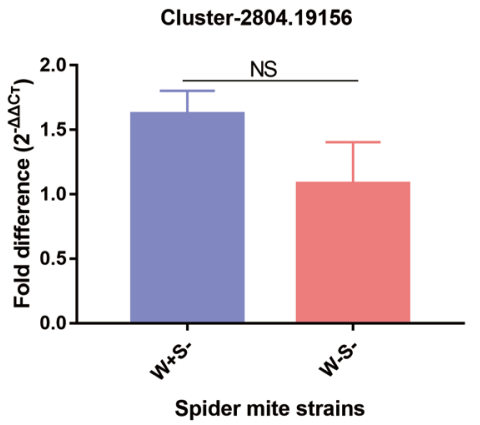

B

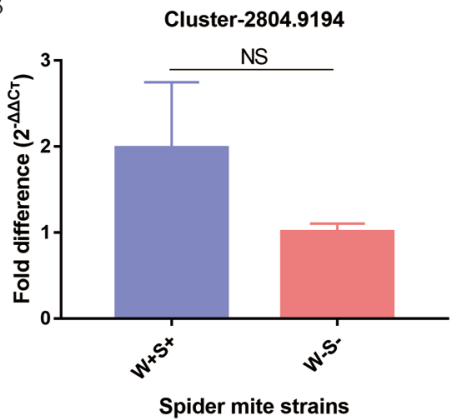

E

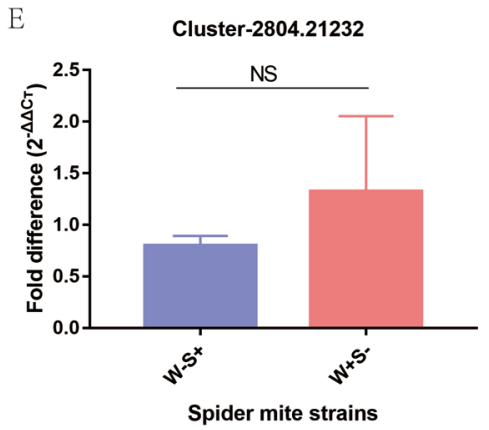

IMB_12607_Figure9.tif

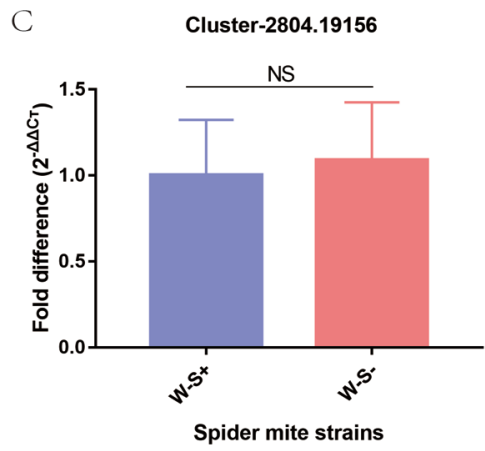

F

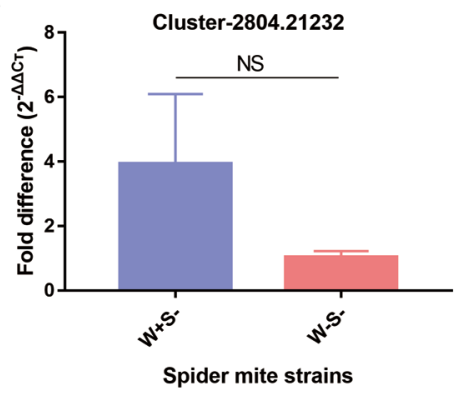

This article is protected by copyright. All rights reserved. 


\section{University Library}

\section{- M M N E R VA A gateway to Melbourne's research publications}

Minerva Access is the Institutional Repository of The University of Melbourne

Author/s:

Yang, K;Xie, K;Zhu, Y-X;Huo, S-M;Hoffmann, A;Hong, X-Y

Title:

Wolbachia dominate Spiroplasma in the co-infected spider mite Tetranychus truncatus

Date:

2019-07-19

Citation:

Yang, K., Xie, K., Zhu, Y. -X., Huo, S. -M., Hoffmann, A. \& Hong, X. -Y. (2019). Wolbachia dominate Spiroplasma in the co-infected spider mite Tetranychus truncatus. INSECT MOLECULAR BIOLOGY, 29 (1), pp.19-37. https://doi.org/10.1111/imb.12607.

Persistent Link:

http://hdl.handle.net/11343/286167 